\title{
TITLE:
}

\section{Entropic formulation of relativistic continuum mechanics}

$\operatorname{AUTHOR}(S):$

Fukuma, Masafumi; Sakatani, Yuho

CITATION:

Fukuma, Masafumi ...[et al]. Entropic formulation of relativistic continuum mechanics. Physical Review E 2011, 84(2): 026315.

ISSUE DATE:

2011-08-15

URL:

http://hdl.handle.net/2433/229551

RIGHT:

(C2011 American Physical Society 
PHYSICAL REVIEW E 84, 026315 (2011)

\title{
Entropic formulation of relativistic continuum mechanics
}

\author{
Masafumi Fukuma* and Yuho Sakatani ${ }^{\dagger}$ \\ Department of Physics, Kyoto University, Kyoto 606-8502, Japan
}

(Received 24 February 2011; published 15 August 2011)

\begin{abstract}
An entropic formulation of relativistic continuum mechanics is developed in the Landau-Lifshitz frame. We introduce two spatial scales, one being the small scale representing the linear size of each material particle and the other the large scale representing the linear size of a large system which consists of material particles and is to linearly regress to the equilibrium. We propose a local functional which is expected to represent the total entropy of the larger system and require the entropy functional to be maximized in the process of linear regression. We show that Onsager's original idea on linear regression can then be realized explicitly as current conservations with dissipative currents in the desired form. We demonstrate the effectiveness of this formulation by showing that one can treat a wide class of relativistic continuum materials, including standard relativistic viscous fluids and relativistic viscoelastic materials.
\end{abstract}

DOI: 10.1103/PhysRevE.84.026315

PACS number(s): 47.75.+f, 05.70.Ln, 46.35.+z, 83.60.Bc

\section{INTRODUCTION}

Thermodynamics is a macroscopic description of a physical system, taking the average of its microscopic degrees of freedom in both the spatial and the temporal directions. The spatial average is the usual coarse graining, while the temporal average is identified with a statistical (ensemble) average (see, e.g., [1]). A global equilibrium of a given system is realized when the temporal average is taken sufficiently longer than any relaxation times of the system. This is characterized as the configuration which maximizes the total entropy. In usual observations, however, the time average is not taken for such a long time, so the materials we often encounter are not in global equilibrium and are regarded as being in the process of regression to equilibrium.

We can say more about this regression to equilibrium if the microscopic interactions of a given system is sufficiently local in both the spatial and the temporal directions. Suppose that we divide the system into subsystems of small size (but still sufficiently large that thermodynamic descriptions work). Then, due to the locality, one may assume that each subsystem gets into equilibrium rapidly. This assumption is called the local equilibrium hypothesis. In the following, when writing space-time coordinates $x=\left(x^{\mu}\right)$ we always assume that dynamical variables are already averaged around $x$ in both the spatial and the temporal directions in such a way that this local equilibrium is realized well with required symmetries being respected. Each spatial unit over which the spatial average is taken is called a material particle.

In the standard description of relativistic fluid mechanics, one adopts the formulation based on conserved currents [2,3] (for recent developments, see [4,5] and references therein). For example, when describing a simple, relativistic fluid in a $(D+1)$-dimensional space-time, one introduces as dynamical variables the energy-momentum tensor $T^{\mu v}(x)$, and the particle-number current (or the charge current) $n^{\mu}(x)$; in all, $(D+1)(D+4) / 2$ degrees of freedom $(=14$ when $D=3)$. They obey the following $D+2$ conservation laws:

$$
\nabla_{\mu} T^{\mu \nu}=0, \quad \nabla_{\mu} n^{\mu}=0 .
$$

\footnotetext{
*fukuma@gauge.scphys.kyoto-u.ac.jp

†yuho@gauge.scphys.kyoto-u.ac.jp
}

Meanwhile, thermodynamic arguments based on the second law of thermodynamics (with a few natural assumptions) tell us that the $(D+1)(D+4) / 2$ fundamental variables can be written only with $D+2$ variables (and their spatial derivatives), and thus the conservations (1) are indeed enough for describing the dynamics of relativistic fluids.

The method described in the previous paragraph is actually powerful and can be applied to a wide class of continuum materials. However, it is rather difficult to see what actually happens entropically in the process of regression to the global minimum. In particular, the link between Onsager's idea of linear regression [6,7] and the current conservations has not been given in a direct manner so far. (An attempt was first made by Casimir [8]. See also [9].)

In this paper, we propose a framework of linear nonequilibrium thermodynamics which directly realizes Onsager's idea of linear regression [6-8] by introducing the explicit form of the entropy functional which is local and to be maximized in the process of thermalization. We show that linear regression to the global equilibrium can be naturally represented in the form of current conservations with dissipative currents in the desired form.

This paper is organized as follows. In Sec. II, we give a relativistic theory of linear nonequilibrium thermodynamics, by closely following the idea of Onsager. We there introduce two spatial scales, $\epsilon_{\mathrm{s}}$ and $L_{\mathrm{s}}$. The smaller scale $\epsilon_{\mathrm{s}}$ represents the linear size of each material particle, while the larger scale $L_{\mathrm{s}}$ represents that of a large system which consists of material particles and is to linearly regress to the equilibrium. We then propose the explicit form of the effective entropy functional and show that Onsager's original idea can be realized explicitly as current conservations with dissipative currents in the desired form. In Sec. III, we demonstrate that usual relativistic fluid mechanics can be reproduced within our framework. In Sec. IV, we apply the formulation to a class of continuum materials [10]. We propose a generally covariant generalization of the theory of viscoelasticity [10-12] and show that the so-called rheology equations given in $[11,12]$ can be naturally obtained in a generally covariant form. Section $\mathrm{V}$ is devoted to a conclusion and discussions. 


\section{GENERAL THEORY}

\section{A. Geometrical setup}

In order to define linear nonequilibrium thermodynamics in a generally covariant manner, we first make a few preparations. We consider continuum materials living in a $(D+1)$ dimensional Lorentzian manifold $\mathcal{M}$. Its local coordinates are denoted by $x^{\mu}(\mu=0,1, \ldots, D)$ and the Lorentzian metric with signature $(-,+, \ldots,+)$ by $g_{\mu v}(x)$. We take the natural unit, $\hbar=c=k_{B}=1$, throughout this paper. In order to develop thermodynamics, we first need to specify a set of time slices (or a foliation) on each of which we consider spatial averages. In this article, we exclusively consider the foliation in which each time slice is orthogonal to the energy flux [or the energy-momentum $(D+1)$-vector] $p_{\mu}$, assuming that $p_{\mu}$ is hypersurface orthogonal. This choice of foliation is called the Landau-Lifshitz frame. We parametrize the hypersurfaces with a real parameter $t$ as $\Sigma_{t}(t \in \mathbb{R})$ and introduce a coordinate system $x=\left(x^{\mu}\right)=\left(x^{0}, \boldsymbol{x}\right)$ such that $x^{0}=t, \boldsymbol{x}=\left(x^{i}\right)(i=1, \ldots, D)$. The unit normal $u^{\mu}(x)$ to the hypersurfaces (called the velocity field) is given by

$$
u^{\mu}(x) \equiv g^{\mu \nu}(x) p_{v}(x) / e(x)=p^{\mu}(x) / e(x),
$$

where $e(x)$ is the density of the proper energy (rest mass plus internal energy),

$$
e(x) \equiv \sqrt{-g^{\mu \nu}(x) p_{\mu}(x) p_{\nu}(x)} .
$$

Here and hereafter, indices are lowered (or raised) always with $g_{\mu \nu}$ (or with its inverse $g^{\mu \nu}$ ). The induced metric on a $D$-dimensional hypersurface passing through $x$ is expressed as

$$
h_{\mu \nu}(x) \equiv g_{\mu \nu}(x)+u_{\mu}(x) u_{\nu}(x)=g_{\mu \nu}(x)+\frac{p_{\mu}(x) p_{\nu}(x)}{e^{2}(x)} .
$$

We define the extrinsic curvature $K_{\mu \nu}$ of the hypersurface as half the Lie derivative of $h_{\mu \nu}$ with respect to the velocity field $u^{\mu}$ :

$$
K_{\mu \nu} \equiv \frac{1}{2} £_{u} h_{\mu \nu}=\frac{1}{2} h_{\mu}^{\rho} h_{\nu}^{\sigma}\left(\nabla_{\rho} u_{\sigma}+\nabla_{\sigma} u_{\rho}\right),
$$

which measures the rate of change in the induced metric $h_{\mu \nu}$ as material particles flow along $u^{\mu}$. Note that this tensor is symmetric and orthogonal to $u^{\mu}, K_{\mu \nu} u^{\nu}=0$.

In the Arnowitt-Deser-Misner (ADM) parametrization [13], the metric and the velocity field are represented with the lapse $N(x)$ and the shifts $N^{i}(x)(i=1, \ldots, D)$ as ${ }^{1}$

$$
\begin{aligned}
d s^{2}= & g_{\mu \nu}(x) d x^{\mu} d x^{\nu}=-N^{2}(x) d t^{2} \\
& +h_{i j}(x)\left[d x^{i}-N^{i}(x) d t\right]\left[d x^{j}-N^{j}(x) d t\right],
\end{aligned}
$$

\footnotetext{
${ }^{1}$ Throughout the present paper, we write a contravariant vector field $v^{\mu}(x)(\mu=0,1, \ldots, D)$ in a concise form as $v=v^{\mu}(x) \partial_{\mu}$, regarding $\partial_{\mu}$ simply as a basis of a vector space (i.e., the tangent space in a mathematical terminology). For example, Eq. (7) stands for the two equations, $u^{0}=1 / N$ and $u^{i}=N^{i} / N(i=1, \ldots, D)$. We denote by $v^{\mu} \nabla_{\mu}$ the covariant derivative along the vector field $v=v^{\mu} \partial_{\mu}$, which now acts on tensor fields as a derivative operator.
}

$$
\begin{gathered}
u=u^{\mu}(x) \partial_{\mu}=\frac{1}{N(x)} \partial_{t}+\frac{N^{i}(x)}{N(x)} \partial_{i} \\
{\left[\Leftrightarrow u_{\mu}(x) d x^{\mu}=-N(x) d t\right] .}
\end{gathered}
$$

With a given foliation, we still have the symmetry of foliation preserving diffeomorphisms that give rise to transformations only among the points on each time slice. Using this residual gauge symmetry we can impose the synchronized gauge, $N^{i}(x) \equiv 0$, so that the background metric and the velocity field become

$$
\begin{gathered}
d s^{2}=g_{\mu \nu}(x) d x^{\mu} d x^{\nu} \equiv-N^{2}(x) d t^{2}+h_{i j}(x) d x^{i} d x^{j}, \\
\left(h_{\mu \nu}\right)=\left(\begin{array}{cc}
0 & 0 \\
0 & h_{i j}
\end{array}\right), \quad\left(h_{\mu}^{\nu}\right)=\left(\begin{array}{cc}
0 & 0 \\
0 & \delta_{i}^{j}
\end{array}\right), \\
u=u^{\mu}(x) \partial_{\mu}=\frac{1}{N(x)} \frac{\partial}{\partial t}=\frac{\partial}{\partial \tau},
\end{gathered}
$$

where $\tau$ is the local proper time defined by $d \tau=N d t$. The volume element on the hypersurface through space-time point $x$ is given by the $D$-form $\sqrt{h} d^{D} \boldsymbol{x}=\sqrt{\operatorname{det}\left(h_{i j}\right)} d^{D} \boldsymbol{x}$. The volume element of the total $(D+1)$-dimensional manifold is given by $\sqrt{-g} d^{D+1} x=N \sqrt{h} d t d^{D} \boldsymbol{x}$. Note that even after taking the synchronized gauge, there remains a residual gauge symmetry of reparametrizing $t$ that labels the time slices.

For generic coordinates (i.e., not necessarily the synchronized coordinates), we introduce the time derivative $D / D t$ as the operation that satisfies the following conditions:

$$
\text { (i) } \frac{D}{D t} t=1, \quad \text { (ii) } \frac{D}{D t} u^{\mu}=0, \quad \text { (iii) Leibniz rule. }
$$

The first condition ensures that $\Delta t(D / D t)$ certainly measures the difference between a quantity on $\Sigma_{t+\Delta t}$ and that on $\Sigma_{t}$. The second condition ensures that if a system of small spatial region can be regarded as being static at time $t$, so can be the system at time $t+\Delta t$. An obvious solution is given by the Lie derivative along the velocity $u=u^{\mu} \partial_{\mu}$ multiplied with the lapse $N$ :

$$
\frac{D}{D t} \equiv N £_{u}
$$

This also satisfies the condition (iii). In the following, the time derivative $D / D t$ will be often abbreviated as the dot.

\section{B. Thermodynamic variables}

As was discussed in Introduction, around each space-time point $x=\left(x^{0}=t, x\right)$ on time slice $\Sigma_{t}$, we make spatial and temporal (or ensemble) averages over $(D+1)$-dimensional regions whose linear sizes we denote by $\epsilon_{\mathrm{s}}$ and $\epsilon_{\mathrm{t}}$, respectively (see Fig. 1). We assume that $\epsilon_{\mathrm{s}}$ and $\epsilon_{\mathrm{t}}$ are both much smaller than the curvature radius of the background metric $g_{\mu \nu}$, so that we can take a local inertial frame at each space-time point $x$ which can be regarded as being flat at least within the extension of $\left(\epsilon_{\mathrm{s}}, \epsilon_{\mathrm{t}}\right)$. This implies that thermodynamic properties of each material particle do not depend on the curvature of the metric when discussing thermodynamics in each material particle. Since the affine connection is not covariant, our assumption means that the covariant local entropy at $x$ depends only on the local value of the metric, $g_{\mu \nu}(x)$.

We assume that the local thermodynamic properties of the material particle at $x$ (already in its local equilibrium) 


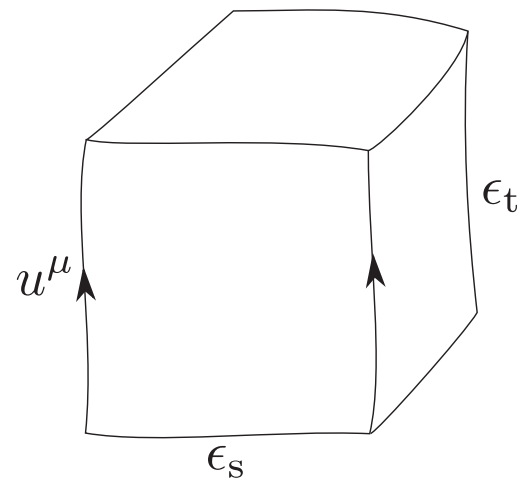

FIG. 1. Local thermodynamic equilibrium.

are specified by the set of local quantities $\left[b^{A}(x), c^{I}(x)\right.$, $\left.d^{P}(x)\right]$. Here $c^{I}(x)$ denote the densities of the existing additive conserved quantities $C^{I} \cdot b^{A}(x)$ denote the "intrinsic" intensive variables possessed by each material particle (such as strains), and $d^{P}(x)$ denote the rest "external" intensive variables which further need to be introduced to characterize each subsystem thermodynamically (such as the background electromagnetic or gravitational fields). In this paper, we distinguish density quantities from other intensive quantities and construct, by multiplying them with the spatial volume element $\sqrt{h}$, new quantities which are spatial densities on each time slice. For example, the entropy density $s$ and the densities $c^{I}$ of conserved charges are density quantities, and for them we construct the following spatial densities:

$$
\tilde{s} \equiv \sqrt{h} s, \quad \tilde{c}^{I} \equiv \sqrt{h} c^{I} .
$$

The local equilibrium hypothesis implies that the local entropy $\tilde{s}(x)$ is already maximized at each space-time point $x$ and is given as a function of the above local variables; $\tilde{s}(x)=$ $\tilde{s}\left(b^{A}(x), \tilde{c}^{I}(x), d^{P}(x)\right)$. This functional relation is sometimes called the fundamental relation in the entropy representation.

In the synchronized gauge, due to the relation $\partial / \partial t=$ $N(x) \partial / \partial \tau$, the proper energy density $e(x)$ measured with the proper time $\tau$ is related to the energy density $\mathrm{e}(x)$ measured with time $t$ as

$$
\mathrm{e}(x)=N(x) e(x) .
$$

Note that $\mathrm{e}(x)$ includes the gravitational potential through the factor $N(x)$. Accordingly, the local temperature $T$ measured with $\tau\left[T \equiv(\partial \tilde{s} / \partial \tilde{e})^{-1}\right]$ is related to the temperature $\mathbf{T}$ measured with $t\left[\mathrm{~T} \equiv(\partial \tilde{s} / \partial \tilde{\mathbf{e}})^{-1}\right]$ through the following Tolman law:

$$
\mathrm{T}(x)=N(x) T(x) .
$$

\section{Entropy functional and the current conservations}

A local thermodynamic equilibrium is realized only in each material particle of spatial size $\epsilon_{\mathrm{s}}$ averaged for a period of time, $\epsilon_{\mathrm{t}}$. If we observe a material at space-time scales larger than $\left(\epsilon_{\mathrm{s}}, \epsilon_{\mathrm{t}}\right)$, we need to take into account the effect that the material particles communicate with each other by exchanging conserved quantities (e.g., the energy-momentum and the particle number). The second law of thermodynamics

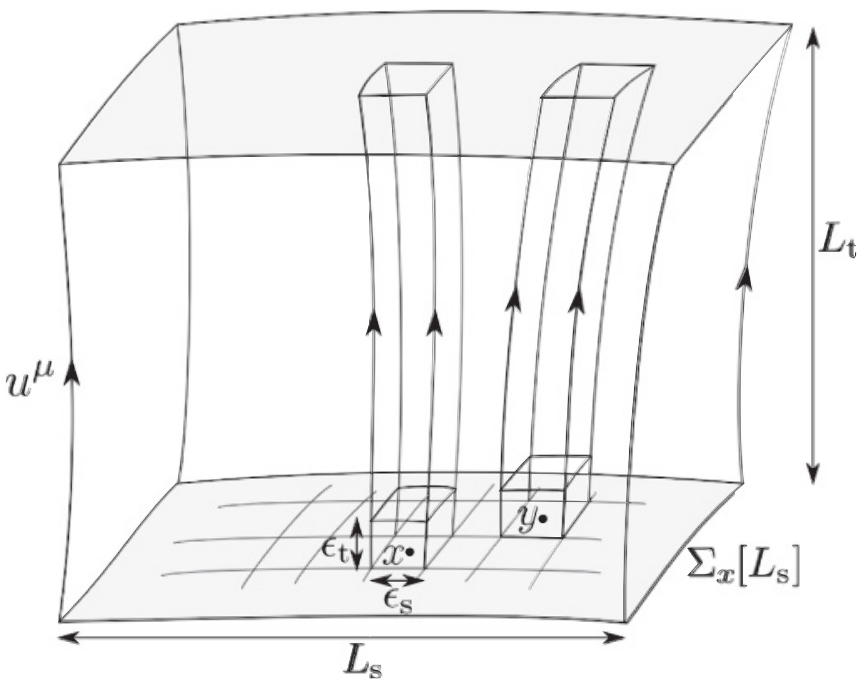

FIG. 2. Time evolution of material particles in the large region $\Sigma_{x}\left[L_{\mathrm{s}}\right]$.

tells us that, if boundary effects can be neglected, this should proceed such that the total entropy of the larger region gets increased.

In order to describe such dynamics mathematically, we first introduce another space-time scale $\left(L_{\mathrm{s}}, L_{\mathrm{t}}\right)$ which is much larger than $\left(\epsilon_{\mathrm{s}}, \epsilon_{\mathrm{t}}\right)$ and assign to each space-time point $x=\left(x^{0}=t, \boldsymbol{x}\right)$ on the time slice $\Sigma_{t}$ a spatial region $\Sigma_{x}\left[L_{\mathrm{s}}\right]$ of linear size $L_{\mathrm{s}}$ (see Fig. 2).

We then consider the total entropy of the region $\Sigma_{x}\left[L_{\mathrm{s}}\right]$ :

$$
\hat{S}\left(t ; \Sigma_{x}\left[L_{\mathrm{s}}\right]\right) \equiv \int_{\Sigma_{x}\left[L_{\mathrm{s}}\right]} d^{D} \boldsymbol{y} \tilde{s}\left(b^{A}(t, \boldsymbol{y}), \tilde{c}^{I}(t, \boldsymbol{y}), d^{P}(t, \boldsymbol{y})\right) .
$$

The irreversible motion of intrinsic variables $a^{r}(x) \equiv$ $\left(b^{A}(x), \tilde{c}^{I}(x)\right)$ at $x$ will proceed toward the equilibrium state of the region $\Sigma_{x}\left[L_{\mathrm{s}}\right]$. Due to the condition $L_{\mathrm{s}} \gg \epsilon_{\mathrm{s}}$, we can assume that the influence from the surroundings of the region $\Sigma_{x}\left[L_{\mathrm{s}}\right]$ is not relevant to the dynamics of $a^{r}(x)$ because $x$ is well inside the region. An equilibrium state of the region $\Sigma_{x}\left[L_{\mathrm{s}}\right]$ will be realized when the observation is made for a long period of time, $L_{\mathrm{t}}$, and can be characterized by the condition

$$
\frac{\delta \hat{S}\left(t ; \Sigma_{x}\left[L_{\mathrm{s}}\right]\right)}{\delta a^{r}(x)}=0 .
$$

Note that the functional derivative is taken only with respect to a spatial, $D$-dimensional unit in the functional. We denote the values of $a^{r}(x)$ at the equilibrium by $a_{0}^{r}\left(x ; L_{\mathrm{s}}\right) \equiv$ $\left(b_{0}^{A}\left(x ; L_{\mathrm{s}}\right), \tilde{c}_{0}^{I}\left(x ; L_{\mathrm{s}}\right)\right)$ and will call the procedure to obtain $a_{0}^{r}\left(x ; L_{\mathrm{s}}\right)$ from $a^{r}(x)$ the dynamical block-spin transformation. One here should note that, since $\tilde{c}^{I}(t, \boldsymbol{y})$ are the densities of conserved quantities, the variations (17) with respect to $\tilde{c}^{I}$-type variables should be taken with total charges kept fixed at prescribed values:

$$
\int_{\Sigma_{x}\left[L_{\mathrm{s}}\right]} d^{D} \boldsymbol{y} \tilde{c}^{I}(t, \boldsymbol{y}) \equiv C^{I}\left(\Sigma_{x}\left[L_{\mathrm{s}}\right]\right) .
$$



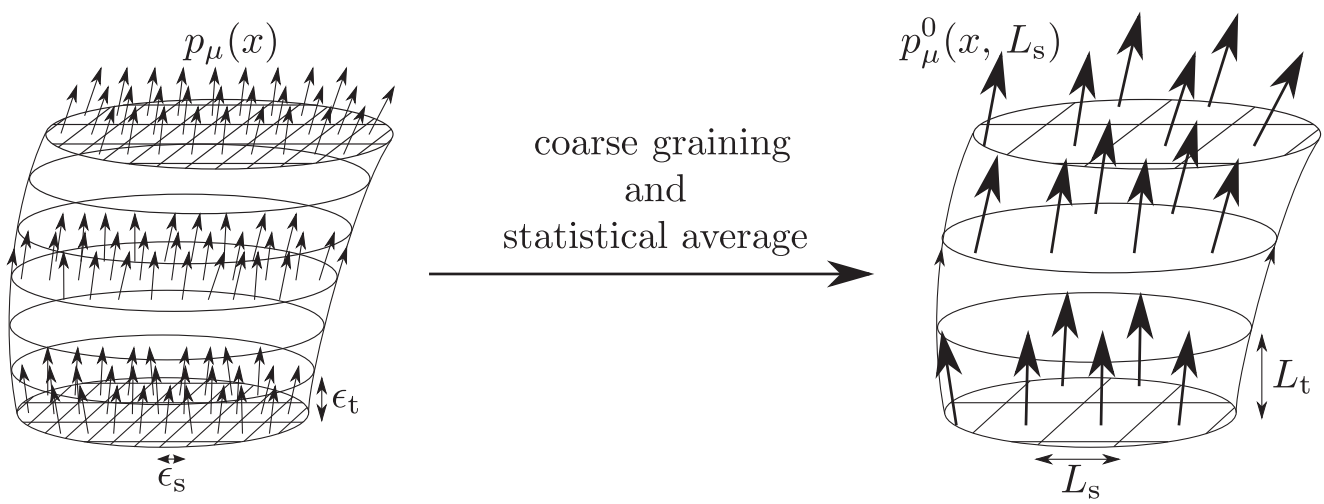

FIG. 3. Dynamical block-spin transformation from the small-scale variable $p_{\mu}(x)$ (left) to the large-scale variable $p_{\mu}^{0}\left(x, L_{\mathrm{s}}\right)($ right $)$.

A simple analysis using the Lagrange multipliers shows that the condition of global equilibrium is expressed locally as

$$
\frac{\partial \tilde{s}}{\partial b^{A}}(x)=0 \quad \text { and } \quad h_{\mu}^{\nu}(x) \nabla_{\nu} \beta_{I}(x)=0,
$$

where $\beta_{I}$ is the thermodynamic variable conjugate to $\tilde{c}^{I}$ that is defined by

$$
\beta_{I}(x) \equiv \frac{\partial \tilde{s}}{\partial \tilde{c}^{I}}(x) .
$$

We here make a few comments. First, the procedure to define the equilibrium values $a_{0}^{r}\left(x ; L_{\mathrm{s}}\right)$ at point $x$ is carried out over its own region $\Sigma_{x}\left[L_{\mathrm{s}}\right]$; for a point $y$ different from $x, a_{0}^{r}\left(y ; L_{\mathrm{s}}\right)$ should be obtained by solving the equation $\delta \hat{S}\left(t ; \Sigma_{y}\left[L_{\mathrm{s}}\right]\right) / \delta a^{r}(y)=0$. However, when $y$ is well inside the region $\Sigma_{x}\left[L_{\mathrm{s}}\right]$, in the approximation of linear regression the value of $a_{0}^{r}\left(y ; L_{\mathrm{s}}\right)$ can be regarded as the same with the value $a_{0}^{r}\left(y ; \Sigma_{x}\left[L_{\mathrm{s}}\right]\right)$ that is obtained by solving the equation $\delta \hat{S}\left(t ; \Sigma_{x}\left[L_{\mathrm{s}}\right]\right) / \delta a^{r}(y)=0$.

The second comment is about the spatial scale $L_{\mathrm{s}}$. We take $L_{\mathrm{s}}$ sufficiently larger than $\epsilon_{\mathrm{s}}$ such that $a_{0}^{r}\left(y ; L_{\mathrm{s}}\right)$ themselves can be treated as thermodynamic variables ${ }^{2}$ as well as that the boundary effects can be safely neglected. At the same time, we also take the space-time scale $\left(L_{\mathrm{s}}, L_{\mathrm{t}}\right)$ not too much larger than $\left(\epsilon_{\mathrm{s}}, \epsilon_{\mathrm{t}}\right)$ such that the values of $a^{r}(y)\left(y=(t, \boldsymbol{y}) \in \Sigma_{x}\left[L_{\mathrm{s}}\right]\right)$ do not differ significantly from those of $a_{0}^{r}\left(x ; L_{\mathrm{s}}\right)$. The latter condition ensures that the fluctuation of $a^{r}(y)$ around $a_{0}^{r}\left(y ; L_{\mathrm{s}}\right)$ can be well approximated by the Gaussian distribution. ${ }^{3}$ So long as $L_{\mathrm{s}}$ is chosen in this way, the time evolution of the local variables $a^{r}(x)$ at time scale $\epsilon_{\mathrm{t}}$ can be analyzed elaborately by decomposing $a^{r}(x)$ into the large-scale variable $a_{0}^{r}(x)$ and the small-scale variable $\left(a-a_{0}\right)^{r}(x)$,

$$
a^{r}(x) \equiv a_{0}^{r}(x)+\left(a-a_{0}\right)^{r}(x) .
$$

\footnotetext{
${ }^{2}$ Their fluctuations can be roughly estimated to be $\Delta a_{0}^{r} /\left\langle a_{0}^{r}\right\rangle \sim$ $\left(\epsilon_{\mathrm{s}} / L_{\mathrm{s}}\right)^{D / 2}$.

${ }^{3} \mathrm{We}$ also require that $\left(L_{\mathrm{s}}, L_{\mathrm{t}}\right)$ be much smaller than the typical scales over which the dynamics may change substantially (such as the typical size of a continuum material or the typical scale of the change in background fields). This ensures that the dependence of $a_{0}^{r}\left(x ; L_{\mathrm{s}}\right)$ on the scale $L_{\mathrm{s}}$ is negligibly mild.
}

In fact, the variables $a_{0}^{r}(x)$ evolve with scale $\left(L_{\mathrm{s}}, L_{\mathrm{t}}\right)$, and their variations at time scale $\epsilon_{\mathrm{t}}$ can actually be regarded as being negligibly small (see Fig. 3). In contrast, the variables $\left(a-a_{0}\right)^{r}(x)$ represent the Gaussian fluctuations in the region $\Sigma_{x}\left[L_{\mathrm{s}}\right]$, and their evolutions can be analyzed with the dynamics of space-time scale $\left(\epsilon_{\mathrm{s}}, \epsilon_{\mathrm{t}}\right)$ in the linear approximation.

We now recall Onsager's discussion on the linear regression of nonequilibrium system [1,6-8]. We first decompose the time derivative of $a^{r}(x), \dot{a}^{r}(x) \equiv D a^{r}(x) / D t$, into the reversible and irreversible parts:

$$
\dot{a}^{r}(x) \equiv\left[\dot{a}^{r}(x)\right]_{\mathrm{rev}}+\left[\dot{a}^{r}(x)\right]_{\mathrm{irr}} .
$$

With the decomposition of $a^{r}(x)$ itself [Eq. (21)], the reversible part can be written as

$$
\left[\dot{a}^{r}(x)\right]_{\mathrm{rev}}=\dot{a}_{0}^{r}(x)+\left[\left(\dot{a}-\dot{a}_{0}\right)^{r}(x)\right]_{\mathrm{rev}},
$$

where the first term describes the reversible, isentropic motion of the large-scale variable $a_{0}^{r}(x)$, and the second represents the part of $\left(\dot{a}-\dot{a}_{0}\right)^{r}(x)$ that does not affect the increase of the total entropy $\hat{S}\left(t ; \Sigma_{x}\left[L_{\mathrm{s}}\right]\right)$. Since in the current discussions $a^{r}(x)$ deviate from the equilibrium values $a_{0}^{r}(x)$ only slightly, we can assume that the irreversible motion of the small-scale variables obeys the linear regression law,

$$
\left[\dot{a}^{r}(x)\right]_{\mathrm{irr}}=\left[\left(\dot{a}-\dot{a}_{0}\right)^{r}(x)\right]_{\mathrm{irr}}=-\gamma_{s}^{r}\left(a-a_{0}\right)^{s}(x) .
$$

Here the matrix $\gamma=\left(\gamma_{s}^{r}\right)$ has only positive eigenvalues, and we have used the relation $\dot{a}_{0}^{r}(x)=\left[\dot{a}_{0}^{r}(x)\right]_{\mathrm{rev}}$, that is, $\left[\dot{a}_{0}^{r}(x)\right]_{\text {irr }}=0$. We then expand the total entropy $\hat{S}\left(t ; \Sigma_{x}\left[L_{\mathrm{s}}\right]\right)$ around $a_{0}^{r}(x)$ to second order and write the difference between the total entropy for the configuration $a^{r}(x), \hat{S}\left(t ; \Sigma_{x}\left[L_{\mathrm{s}}\right]\right)=\int_{\Sigma_{x}\left[L_{\mathrm{s}}\right]} d^{D} \boldsymbol{y} \tilde{\boldsymbol{s}}\left(b^{A}(t, \boldsymbol{y}), \tilde{c}^{I}(t, \boldsymbol{y}), d^{P}(t, \boldsymbol{y})\right)$, and that at the equilibrium $a_{0}^{r}(x), \quad \hat{S}_{0}\left(t ; \Sigma_{x}\left[L_{\mathrm{s}}\right]\right) \equiv$ $\int_{\Sigma_{x}\left[L_{\mathrm{s}}\right]} d^{D} \boldsymbol{y} \tilde{\boldsymbol{s}}\left(b_{0}^{A}(t, \boldsymbol{y}), \tilde{c}_{0}^{I}(t, \boldsymbol{y}), d^{P}(t, \boldsymbol{y})\right)$, as

$$
\begin{aligned}
\Delta \hat{S}\left(t ; \Sigma_{x}\left[L_{\mathrm{s}}\right]\right) & \equiv \hat{S}\left(t ; \Sigma_{x}\left[L_{\mathrm{s}}\right]\right)-\hat{S}_{0}\left(t ; \Sigma_{x}\left[L_{\mathrm{s}}\right]\right) \\
& =-\frac{1}{2} \int_{\Sigma_{x}\left[L_{\mathrm{s}}\right]} d^{D} \boldsymbol{y}\left(a-a_{0}\right)^{r} \beta_{r s}\left(a-a_{0}\right)^{s} .
\end{aligned}
$$


Here $\beta_{r s}$ is a symmetric, positive semidefinite operator (which may include spatial derivative operators). The thermodynamic forces are then found to be

$$
f_{r}(x) \equiv \frac{\delta \Delta \hat{S}\left(t ; \Sigma_{x}\left[L_{s}\right]\right)}{\delta a^{r}(x)}=-\beta_{r s}\left(a-a_{0}\right)^{s}(x),
$$

and thus, substituting them into Eq. (24), we obtain the equations of linear regression:

$$
\begin{aligned}
{\left[\dot{a}^{r}(x)\right]_{\text {irr }} } & =L^{r s} f_{s}(x) \\
& =L^{r s} \frac{\delta \Delta \hat{S}\left(t ; \Sigma_{x}\left[L_{\mathrm{s}}\right]\right)}{\delta a^{s}(x)}\left[L^{r s} \equiv\left(\gamma \beta^{-1}\right)^{r s}\right] .
\end{aligned}
$$

The so-called phenomenological coefficients $L^{r s}$ can be shown to satisfy Onsager's reciprocal relation [6-8]

$$
L^{r s}=(-1)^{\left|a^{r}\right|+\left|a^{s}\right|} L^{s r},
$$

where the index $\left|a^{r}\right|$ expresses how the variables transform under time reversal, $a^{r}(x) \rightarrow(-1)^{\left|a^{r}\right|} a^{r}(x){ }^{4}$ The Curie principle says that $L^{r s}$ can be block diagonalized with respect to the transformation properties of the indices $(r, s)$ under spatial rotations and the parity transformation [9], that is, under the subgroup $\mathrm{O}(D)$ of the local Lorentz group $\mathrm{O}(D, 1)$ in local inertial frames. For example, when $a^{r}$ constitute a contravariant vector, $\left(a^{r}\right) \equiv\left(a^{\mu}\right)$, the equations of linear regression should be set for each of the normal and tangential components to the time slice through $x$ :

$$
\begin{aligned}
& {[\dot{a}(x)]_{\operatorname{irr} \perp}^{\mu}(x)=L_{\perp}^{\mu \nu}\left[\frac{\delta \Delta \hat{S}}{\delta a^{v}(x)}\right]_{\perp},} \\
& {[\dot{a}(x)]_{\operatorname{irr} \|}^{\mu}(x)=L_{\|}^{\mu \nu}\left[\frac{\delta \Delta \hat{S}}{\delta a^{v}(x)}\right]_{\|},}
\end{aligned}
$$

where for a contravariant vector $v^{\mu}$ we define $v_{\perp}^{\mu} \equiv\left(-u^{\mu} u_{v}\right) v^{v}$ and $v_{\|}^{\mu} \equiv h_{\nu}^{\mu} v^{v}$ (and similarly for covariant vectors). Covariance and positivity further imposes the condition that $L_{\perp}^{\mu \nu}$ and $L_{\|}^{\mu \nu}$ should be expressed as $L_{\perp}^{\mu \nu}=L_{\perp} u^{\mu} u^{\nu}\left(L_{\perp}>0\right)$ and $L_{\|}^{\mu \nu}=L_{\|} h^{\mu \nu}\left(L_{\|}>0\right)$, respectively.

In Eq. (25), the total entropy $\hat{S}\left(t ; \Sigma_{x}\left[L_{\mathrm{s}}\right]\right)$ is expanded around the equilibrium states $a_{0}^{r}(y)\left(y \in \Sigma_{x}\left[L_{\mathrm{s}}\right]\right)$ as a quadratic form in $\left(a-a_{0}\right)^{r}(y)$. However, due to the equilibrium condition (19), it should be more efficient to express it as a quadratic form in $\left(b-b_{0}\right)^{A}(y)$ and $h_{\mu}^{\nu} \nabla_{\nu} \beta_{I}(y)$, because we then can ignore the subtleness existing in the constrained variations with respect to $\tilde{c}^{I}$-type variables. This consideration leads us to propose that the total entropy can be effectively expressed by the following local functional (to be called the entropy

\footnotetext{
${ }^{4}$ When the system is subject to external fields $\boldsymbol{H}$ which change the sign under time reversal (like magnetic field), the reciprocal relation is expressed as $L^{r s}(\boldsymbol{H})=(-1)^{\left|a^{r}\right|+\left|a^{s}\right|} L^{s r}(-\boldsymbol{H})$.
}

functional):

$$
\begin{aligned}
& \hat{S}\left(t ; \Sigma_{x}\left[L_{\mathrm{s}}\right]\right) \\
& \equiv \hat{S}_{0}\left(t ; \Sigma_{x}\left[L_{\mathrm{s}}\right]\right)-\frac{1}{2} \int_{\Sigma_{x}\left[L_{\mathrm{s}}\right]} d^{D} \boldsymbol{y} N \sqrt{h} \\
& \times\left(\left(b-b_{0}\right)^{A} \nabla_{\mu} \beta_{I}\right)\left(\begin{array}{cc}
\ell_{A B} & \ell_{A}^{\nu J} \\
\ell_{B}^{\mu I} & \ell^{\mu I, \nu J}
\end{array}\right)\left(\begin{array}{c}
\left(b-b_{0}\right)^{B} \\
\nabla_{\nu} \beta_{J}
\end{array}\right) \\
& \quad\left(\ell_{A}^{\mu I} u_{\mu}=0=\ell^{\mu I, \nu J} u_{\nu}\right) .
\end{aligned}
$$

Here the coefficient $\left(\begin{array}{cc}\ell_{A B} & \ell_{A}^{\nu J} \\ \ell_{B}^{\mu I} & \ell^{\mu I, v J}\end{array}\right)$ is a symmetric, positive semidefinite matrix. We have extracted the factor $N$ from the coefficient matrices for later convenience (recall that $N \sqrt{h}=$ $\sqrt{-g}$ ). The matrix elements in principle could be calculated from the fundamental relation, $\tilde{s}=\tilde{s}\left(b^{A}, \tilde{c}^{I}, d^{P}\right)$, once the foliation is fixed [see the Appendix for the derivation of (31) for simple cases]. However, as we see below, their explicit forms need not be specified for the following discussions.

With the entropy functional (31), the thermodynamic forces in the equations of linear regression Eq. (27) are calculated as

$$
\begin{aligned}
f_{A}=\frac{\delta \Delta \hat{S}}{\delta b^{A}}= & -\sqrt{-g}\left[\ell_{A B}\left(b-b_{0}\right)^{B}+\ell_{A}^{\mu I} \nabla_{\mu} \beta_{I}\right. \\
& \left.-s_{A I} \nabla_{\mu}\left(\ell_{B}^{\mu I}\left(b-b_{0}\right)^{B}+\ell^{\mu I, \nu J} \nabla_{\nu} \beta_{J}\right)\right] \\
\simeq & -\sqrt{-g}\left[\ell_{A B}\left(b-b_{0}\right)^{B}+\ell_{A}^{\mu I} \nabla_{\mu} \beta_{I}\right] \\
f_{I}=\frac{\delta \Delta \hat{S}}{\delta \tilde{c}^{I}}= & \sqrt{-g}\left(\frac{\partial^{2} \tilde{s}}{\partial \tilde{c}^{I} \partial \tilde{c}^{J}}\right) \\
& \times \nabla_{\mu}\left[\ell_{B}^{\mu J}\left(b-b_{0}\right)^{B}+\ell^{\mu J, \nu K} \nabla_{\nu} \beta_{K}\right] \\
\simeq & N s_{I J}^{0} \nabla_{\mu}\left[\ell_{B}^{\mu J}\left(b-b_{0}\right)^{B}+\ell^{\mu J, \nu K} \nabla_{\nu} \beta_{K}\right]
\end{aligned}
$$

where $s_{A I} \equiv \partial^{2} \tilde{s} / \partial b^{A} \partial \tilde{c}^{I}, s_{I J} \equiv \sqrt{h} \partial^{2} \tilde{s} / \partial \tilde{c}^{I} \partial \tilde{c}^{J}$, and $s_{I J}^{0}$ is the value of $s_{I J}$ at the equilibrium. In obtaining the last line of Eq. (32) we have neglected higher-order terms in the derivative expansion. Then the irreversible evolution of $\left(b^{A}, \tilde{c}^{I}\right)$ is given by

$$
\begin{aligned}
{[\dot{b}]_{\mathrm{irr}}^{A} } & =\frac{1}{\sqrt{h}} L^{A B} f_{B}+L^{A I} f_{I}, \\
{[\dot{\tilde{c}}]_{\mathrm{irr}}^{I} } & =L^{I A} f_{A}+\sqrt{h} L^{I J} f_{J},
\end{aligned}
$$

where we have multiplied $(\sqrt{h})^{ \pm 1}$ to make $L^{r s}$ tensors (not tensor densities). By further assuming that $L^{I A}=L^{A I}=0$ and by using Eqs. (32) and (33), the above equations can be rewritten as

$$
\begin{aligned}
\dot{b}^{A}-[\dot{b}]_{\mathrm{rev}}^{A} & =[\dot{b}]_{\mathrm{irr}}^{A} \\
& =-N L^{A B}\left[\ell_{B C}\left(b-b_{0}\right)^{C}+\ell_{B}^{\mu I} \nabla_{\mu} \beta_{I}\right], \\
\dot{\tilde{c}}^{I}-[\dot{\tilde{c}}]_{\mathrm{rev}}^{I}= & {[\dot{\tilde{c}}]_{\mathrm{irr}}^{I} } \\
= & \sqrt{-g} L^{I J} s_{J K}^{0} \nabla_{\mu}\left[\ell_{B}^{\mu K}\left(b-b_{0}\right)^{B}+\ell^{\mu K, \nu L} \nabla_{\nu} \beta_{L}\right] .
\end{aligned}
$$

The coefficient $L^{I J} s_{J K}^{0}$ can be regarded as being constant in our derivative expansion, and, as we see in the next section, for the cases of interest the quantities $\dot{\tilde{c}}^{I}=N £_{u}\left(\sqrt{h} c^{I}\right)$ can be 
always written as $\sqrt{-g} \nabla_{\mu}\left(c^{I} u^{\mu}\right)$. We thus can rewrite Eq. (37) in the form of current conservations:

$$
\nabla_{\mu} J^{I \mu}=0 .
$$

Here the current $J^{I \mu}$ consists of three parts:

$$
J^{I \mu}=c^{I} u^{\mu}+J_{(\mathrm{r})}^{I \mu}+J_{(\mathrm{d})}^{I \mu} .
$$

The first is the convective part, and the third is the dissipative part defined by

$$
J_{(\mathrm{d})}^{I \mu} \equiv-L^{I J} s_{J K}^{0}\left[\ell_{B}^{\mu K}\left(b-b_{0}\right)^{B}+\ell^{\mu K, \nu L} \nabla_{\nu} \beta_{L}\right] .
$$

The second comes from the equation $[\dot{\tilde{c}}]_{\mathrm{rev}}^{I}=-\sqrt{-g} \nabla_{\mu} J_{(\mathrm{r})}^{I \mu}$, which should be derived from another theory describing reversible, isentropic processes. For example, for simple viscous fluids, such theory should be that of ideal fluids.

When there are only $c^{I}$-type variables which are all scalar functions, the coefficients $\ell^{\mu I, \nu J}$ should take the form $\ell^{\mu I, \nu J}=$ $-\ell h^{\mu \nu}\left(s_{0}^{-1}\right)^{I J}(\ell \geqslant 0)$ due to the positivity and the covariance, and the dissipative current is expressed as

$$
J_{(\mathrm{d})}^{I \mu} \equiv \ell L^{I J} h^{\mu \nu} \nabla_{\nu} \beta_{J}=\ell L^{I J} h^{\mu \nu} \nabla_{\nu}\left(\frac{\partial \tilde{s}}{\partial \tilde{c}^{J}}\right) .
$$

The second law of thermodynamics says that $\hat{S}\left(t ; \Sigma_{x}\left[L_{\mathrm{s}}\right]\right)$ is a monotonically increasing function of time $t$. Indeed, the entropy production rate $P\left(t ; \Sigma_{x}\left[L_{\mathrm{s}}\right]\right)$ in the region $\Sigma_{x}\left[L_{\mathrm{s}}\right]$ is always positive for time scale $\epsilon_{\mathrm{t}}$ due to the positivity of the phenomenological coefficients:

$$
\begin{aligned}
P\left(t ; \Sigma_{x}\left[L_{\mathrm{s}}\right]\right) & \equiv \frac{D}{D t} \Delta S\left(t ; \Sigma_{x}\left[L_{\mathrm{s}}\right]\right) \\
& =\int_{\Sigma_{x}\left[L_{\mathrm{s}}\right]} d^{D} \boldsymbol{y} \frac{\delta \Delta \hat{S}}{\delta a^{r}} \dot{a}^{r} \\
& =\int_{\Sigma_{x}\left[L_{\mathrm{s}}\right]} d^{D} \boldsymbol{y} \frac{\delta \Delta \hat{S}}{\delta a^{r}}\left[\dot{a}^{r}\right]_{\mathrm{irr}} \\
& =\int_{\Sigma_{x}\left[L_{\mathrm{s}}\right]} d^{D} \boldsymbol{y} L^{r s} \frac{\delta \Delta \hat{S}}{\delta a^{r}} \frac{\delta \Delta \hat{S}}{\delta a^{s}} \geqslant 0 .
\end{aligned}
$$

Here we have neglected the contributions from $\dot{d}^{P}$ because they would be of higher orders. We also have used the identity $\int_{\Sigma_{x}\left[L_{\mathrm{s}}\right]} d^{D} \boldsymbol{y}\left(\delta \Delta \hat{S} / \delta a^{r}\right)\left[\dot{a}^{r}\right]_{\text {rev }}=0$. One can further show that the entropy production is a monotonically decreasing function when $\left[\dot{a}^{r}\right]_{\mathrm{rev}}=0 ; \dot{P}\left(t ; \Sigma_{x}\left[L_{\mathrm{s}}\right]\right) \leqslant 0$.

\section{RELATIVISTIC FLUID MECHANICS}

\section{A. Relativistic fluids in the Landau-Lifshitz frame}

We define relativistic fluids as continuum materials whose thermodynamic properties can be characterized only by the local energy-momentum $\tilde{p}_{\alpha}=\sqrt{h} p_{\alpha}(\alpha=0,1, \ldots, D)$, the local particle number $\tilde{n}=\sqrt{h} n$, and the background metric $g_{\mu \nu}$.

To relativistic fluids, the formalism in the previous section can be applied with the following identifications:

\begin{tabular}{cccccc}
$b^{A}$ & $\left(\partial \tilde{s} / \partial b^{A}\right)$ & $\tilde{c}^{I}$ & $\beta_{I}$ & $d^{P}$ & $\left(\partial \tilde{s} / \partial d^{P}\right)$ \\
\hline Nothing & Nothing & $\tilde{p}_{\alpha}$ & $-u^{\alpha} / T$ & $g_{\mu \nu}$ & $\sqrt{h} T_{(\mathrm{q})}^{\mu \nu} / 2 T$ \\
& & $\tilde{n}$ & $-\mu / T$ & &
\end{tabular}

We here explain the entities in the list. We shall assume that $\tilde{s}$ depends on $\tilde{p}_{\alpha}$ only through the local energy $\tilde{e}\left(\tilde{p}_{\alpha}, g_{\mu \nu}\right)=$ $\sqrt{-g^{\mu v} \tilde{p}_{\mu} \tilde{p}_{v}}$ so that the fundamental relation is expressed in the following form:

$$
\tilde{s}\left(\tilde{p}_{\alpha}, \tilde{n}, g_{\mu \nu}\right)=\tilde{\sigma}\left(\tilde{e}\left(\tilde{p}_{\alpha}, g_{\mu \nu}\right), \tilde{n}, g_{\mu \nu}\right) .
$$

Then, by introducing the temperature $T$, the chemical potential $\mu$ and the quasiconservative stress $\tau_{(\mathrm{q})}^{\mu \nu}$ as

$$
\begin{gathered}
\frac{\partial \tilde{\sigma}\left(\tilde{e}, \tilde{n}, g_{\mu \nu}\right)}{\partial \tilde{e}} \equiv \frac{1}{T}, \quad \frac{\partial \tilde{\sigma}\left(\tilde{e}, \tilde{n}, g_{\mu \nu}\right)}{\partial \tilde{n}} \equiv-\frac{\mu}{T}, \\
\frac{\partial \tilde{\sigma}\left(\tilde{e}, \tilde{n}, g_{\mu \nu}\right)}{\partial g_{\mu \nu}} \equiv \frac{\sqrt{h}}{2 T} \tau_{(\mathrm{q})}^{\mu \nu},
\end{gathered}
$$

and by using the identities

$$
\frac{\partial \tilde{e}}{\partial \tilde{p}_{\alpha}}=-\frac{\tilde{p}^{\alpha}}{\tilde{e}}=-u^{\alpha}, \quad \frac{\partial \tilde{e}}{\partial g_{\mu \nu}}=\frac{\tilde{p}^{\mu} \tilde{p}^{v}}{2 \tilde{e}}=\frac{\tilde{e} u^{\mu} u^{\nu}}{2},
$$

the variation of the local entropy $\tilde{s}$ is expressed as

$$
\delta \tilde{s}=-\frac{u^{\alpha}}{T} \delta \tilde{p}_{\alpha}-\frac{\mu}{T} \delta \tilde{n}+\frac{\sqrt{h}}{2 T} T_{(\mathrm{q})}^{\mu \nu} \delta g_{\mu \nu},
$$

where $T_{(\mathrm{q})}^{\mu v}$ is the quasiconservative energy-momentum tensor:

$$
T_{(\mathrm{q})}^{\mu \nu} \equiv e u^{\mu} u^{\nu}+\tau_{(\mathrm{q})}^{\mu \nu}=p^{\mu} u^{\nu}+\tau_{(\mathrm{q})}^{\mu \nu} .
$$

In order to make this decomposition unique, we require that $\tau_{(\mathrm{q})}^{\mu \nu}$ be orthogonal to $u^{\mu}$ :

$$
\tau_{(\mathrm{q})}^{\mu \nu} u_{v}=0
$$

Simple fluids (that have no specific spatial directions) are realized by taking $\tau_{(\mathrm{q})}^{\mu \nu}=P h^{\mu \nu}$ with $P$ the pressure. Then, by using the identity $h^{\mu v} \delta g_{\mu \nu}=h^{\mu v} \delta h_{\mu \nu}=$ $2 \delta \sqrt{h} / \sqrt{h}$, Eq. (47) becomes the standard expression for the local entropy $\tilde{s}=\tilde{\sigma}\left(\tilde{e}, \tilde{n}, g_{\mu \nu}\right)$ of simple fluids, $\delta \tilde{s}=$ $\delta \tilde{\sigma}=(1 / T) \delta \tilde{e}-(\mu / T) \delta \tilde{n}+P \delta \sqrt{h} ;$ or equivalently, $\delta s=$ $\delta(\tilde{s} / \sqrt{h})=(1 / T) \delta e-(\mu / T) \delta n$.

As for the entropy functional, from the general expression (31) without $b^{A}$-type variables, we have

$$
\begin{aligned}
\Delta \hat{S} \equiv & -\frac{1}{2} \int_{\Sigma_{x}\left[L_{s}\right]} d^{D} \boldsymbol{y} N \sqrt{h}\left[\ell_{\alpha, \beta}^{\mu \nu} \nabla_{\mu}\left(\frac{\partial \tilde{s}}{\partial \tilde{p}_{\alpha}}\right) \nabla_{\nu}\left(\frac{\partial \tilde{s}}{\partial \tilde{p}_{\beta}}\right)\right. \\
& \left.+m^{\mu \nu} \partial_{\mu}\left(\frac{\partial \tilde{s}}{\partial \tilde{n}}\right) \partial_{\nu}\left(\frac{\partial \tilde{s}}{\partial \tilde{n}}\right)\right] .
\end{aligned}
$$

We assume that the coefficient functions $\ell_{\alpha, \beta}^{\mu \nu}$ and $m^{\mu \nu}$ are all orthogonal to the velocity field; $\ell_{\alpha, \beta}^{\mu}{ }_{\nu} u_{v}=0, \ell_{\alpha, \beta}^{\mu}{ }_{\nu}^{v} u^{\beta}=0$, and $m^{\mu v} u_{v}=0$, and also that the second derivatives of the form $\partial^{2} \tilde{s} / \partial \tilde{p}_{\alpha} \partial \tilde{n}$ are small and can be neglected. The maximum of the entropy functional is then given by $\left(p_{\alpha}^{0}, n^{0}\right)$ that satisfy the following equations:

$$
\begin{aligned}
\left.h^{\mu \nu} h_{\alpha \beta} \nabla_{\nu}\left(\frac{\partial \tilde{s}}{\partial \tilde{p}_{\beta}}\right)\right|_{0} & =\left.h^{\mu \nu} h_{\alpha \beta} \nabla_{\nu}\left(-\frac{u^{\beta}}{T}\right)\right|_{0} \\
& =-\left.\frac{1}{T} h^{\mu \nu} h_{\alpha \beta} \nabla_{\nu} u^{\beta}\right|_{0}=0 \\
\left.h^{\mu \nu} \partial_{\nu}\left(\frac{\partial \tilde{s}}{\partial \tilde{n}}\right)\right|_{0} & =\left.h^{\mu \nu} \partial_{\nu}\left(-\frac{\mu}{T}\right)\right|_{0}=0 .
\end{aligned}
$$


Note that these equations hold only within the spatial region $\Sigma_{x}\left[L_{\mathrm{s}}\right]$.

The variation of the entropy functional (50) is given by

$$
\begin{aligned}
\frac{\delta \Delta \hat{S}}{\delta \tilde{p}_{\lambda}} & =\sqrt{-g} \frac{\partial^{2} \tilde{s}}{\partial \tilde{p}_{\lambda} \partial \tilde{p}_{\alpha}} \nabla_{\mu}\left[\ell_{\alpha, \beta}^{\mu \nu} \nabla_{\nu}\left(\frac{\partial \tilde{s}}{\partial \tilde{p}_{\beta}}\right)\right] \\
& =\sqrt{-g} \frac{\partial^{2} \tilde{s}}{\partial \tilde{p}_{\lambda} \partial \tilde{p}_{\alpha}} \nabla_{\mu}\left[-\frac{1}{T} \ell_{\alpha, \beta}^{\mu \nu} \nabla_{\nu} u^{\beta}\right], \\
\frac{\delta \Delta \hat{S}}{\delta \tilde{n}} & =\sqrt{-g} \frac{\partial^{2} \tilde{s}}{\partial \tilde{n}^{2}} \nabla_{\mu}\left[m^{\mu \nu} \partial_{\nu}\left(\frac{\partial \tilde{s}}{\partial \tilde{n}}\right)\right] \\
& =\sqrt{-g} \frac{\partial^{2} \tilde{s}}{\partial \tilde{n}^{2}} \nabla_{\mu}\left[m^{\mu \nu} \partial_{\nu}\left(-\frac{\mu}{T}\right)\right] .
\end{aligned}
$$

By using the decomposition of the matrix $\partial^{2} \tilde{s} / \partial \tilde{p}_{\mu} \partial \tilde{p}_{\nu}$ (negative-definite for each irreducible component) as

$$
\begin{gathered}
\sqrt{h} \frac{\partial^{2} \tilde{s}}{\partial \tilde{p}_{\mu} \partial \tilde{p}_{v}}=-c_{\perp} u^{\mu} u^{v}-c_{\|} h^{\mu \nu} \\
\left(c_{\perp} \equiv-\sqrt{h} \frac{\partial^{2} \tilde{s}}{\partial \tilde{e}^{2}}(>0), \quad c_{\|} \equiv \frac{1}{e T}(>0)\right),
\end{gathered}
$$

the vector $\delta \Delta \hat{S} / \delta \tilde{p}_{\lambda}$ is decomposed as

$$
\frac{\delta \Delta \hat{S}}{\delta \tilde{p}_{\lambda}}=\left[\frac{\delta \Delta \hat{S}}{\delta \tilde{p}_{\lambda}}\right]_{\perp}+\left[\frac{\delta \Delta \hat{S}}{\delta \tilde{p}_{\lambda}}\right]_{\|},
$$

with

$$
\begin{aligned}
{\left[\frac{\delta \Delta \hat{S}}{\delta \tilde{p}_{\lambda}}\right]_{\perp} } & \equiv\left(-u^{\lambda} u^{\nu}\right) \frac{\delta \Delta \hat{S}}{\delta \tilde{p}_{\nu}} \\
& =N c_{\perp}\left(-u^{\lambda} u^{\nu}\right) \nabla_{\mu}\left[-\frac{1}{T} \ell_{\nu, \sigma}^{\mu \rho} \nabla_{\rho} u^{\sigma}\right], \\
{\left[\frac{\delta \Delta \hat{S}}{\delta \tilde{p}_{\lambda}}\right]_{\|} } & \equiv h^{\lambda \nu} \frac{\delta \Delta \hat{S}}{\delta \tilde{p}_{v}}=-N c_{\|} h^{\lambda \nu} \nabla_{\mu}\left[-\frac{1}{T} \ell_{\nu, \sigma}^{\mu \rho} \nabla_{\rho} u^{\sigma}\right] .
\end{aligned}
$$

With the variations of the entropy functional Eqs. (54), (57), and (58), we set the following equations of linear regression:

$$
\begin{gathered}
{\left[\dot{\tilde{p}}_{\alpha}-\left[\dot{\tilde{p}}_{\alpha}\right]_{\mathrm{rev}}\right]_{\perp}=\left[\dot{\tilde{p}}_{\alpha}\right]_{\mathrm{irr} \perp}=\sqrt{h} L_{\perp} u_{\alpha} u_{\lambda}\left[\frac{\delta \Delta \hat{S}}{\delta \tilde{p}_{\lambda}}\right]_{\perp},} \\
{\left[\dot{\tilde{p}}_{\alpha}-\left[\dot{\tilde{p}}_{\alpha}\right]_{\mathrm{rev}}\right]_{\|}=\left[\dot{\tilde{p}}_{\alpha}\right]_{\mathrm{irr} \|}=\sqrt{h} L_{\|} h_{\alpha \lambda}\left[\frac{\delta \Delta \hat{S}}{\delta \tilde{p}_{\lambda}}\right]_{\|},} \\
\dot{\tilde{n}}-[\dot{\tilde{n}}]_{\mathrm{rev}}=[\dot{\tilde{n}}]_{\mathrm{irr}} \equiv \sqrt{h} M \frac{\delta \Delta \hat{S}}{\delta \tilde{n}} .
\end{gathered}
$$

Note that the time derivatives of $\tilde{p}_{\alpha}$ and $\tilde{n}$ are given by

$$
\begin{aligned}
\frac{D}{D t} \tilde{p}_{\alpha} & \equiv N £_{u} \tilde{p}_{\alpha}=\sqrt{-g} \nabla_{\mu}\left(p_{\alpha} u^{\mu}\right), \\
\frac{D}{D t} \tilde{n} & \equiv N £_{u} \tilde{n}=\sqrt{-g} \nabla_{\mu}\left(n u^{\mu}\right) .
\end{aligned}
$$

Equations (62) and (63) can be shown by using the identities $£_{u} \sqrt{h}=\sqrt{h} \nabla_{\mu} u^{\mu}$ and $p_{\mu} \nabla_{\alpha} u^{\mu}=0$. The former identity can be proved as $£_{u} \sqrt{h}=(1 / 2) \sqrt{h} h^{\mu \nu} £_{u} h_{\mu \nu}=(1 / 2)$ $\sqrt{h} h^{\mu \nu} £_{u} g_{\mu \nu}=(1 / 2) \sqrt{h} h^{\mu \nu}\left(\nabla_{\mu} u_{\nu}+\nabla_{\nu} u_{\mu}\right)=(1 / 2) \sqrt{h} g^{\mu \nu}$ $\left(\nabla_{\mu} u_{v}+\nabla_{\nu} u_{\mu}\right)=\sqrt{h} \nabla_{\mu} u^{\mu}$. The latter follows from the facts that $p_{\mu}=e u_{\mu}$ and $u^{\mu} u_{\mu}=-1$. One can then easily show that the left-hand side of Eqs. (59)-(61) can be rewritten as

$$
\begin{aligned}
{\left[\dot{\tilde{p}}_{\alpha}-\left[\dot{\tilde{p}}_{\alpha}\right]_{\mathrm{rev}}\right]_{\perp} } & =\left(-u_{\alpha} u^{v}\right)\left[\sqrt{-g} \nabla_{\mu}\left(p_{\nu} u^{\mu}\right)-\left[\dot{\tilde{p}}_{v}\right]_{\mathrm{rev}}\right] \\
{\left[\dot{\tilde{p}}_{\alpha}-\left[\dot{\tilde{p}}_{\alpha}\right]_{\mathrm{rev}}\right]_{\|} } & =h_{\alpha}^{v}\left[\sqrt{-g} \nabla_{\mu}\left(p_{\nu} u^{\mu}\right)-\left[\dot{\tilde{p}}_{\nu}\right]_{\mathrm{rev}}\right] \\
\dot{\tilde{n}}-[\dot{\tilde{n}}]_{\mathrm{rev}} & =\sqrt{-g} \nabla_{\mu}\left(n u^{\mu}\right)-[\dot{\tilde{n}}]_{\mathrm{rev}}
\end{aligned}
$$

Furthermore, one expects that the reversible (or isentropic) evolutions of $\tilde{p}_{\mu}$ and $\tilde{n}$ can be identified as the evolutions for ideal fluids,

$$
\left[\dot{\tilde{p}}_{\alpha}\right]_{\mathrm{rev}}=-\sqrt{-g} \nabla_{\mu} \tau_{\alpha}^{(\mathrm{r}) \mu}, \quad[\dot{\tilde{n}}]_{\mathrm{rev}}=0 .
$$

The isentropic current $\tau_{\alpha}^{(\mathrm{r}) \mu}$ equals the quasiconservative stress tensor $\tau_{\alpha}^{(\mathrm{q})} \mu$, as is shown in the next section. Then Eqs. (59)(61) become

$$
\begin{aligned}
\left(-u_{\alpha} u^{\nu}\right) \nabla_{\mu} T_{\nu}^{(\mathrm{q}) \mu} & =-\left(-u_{\alpha} u^{\nu}\right) c_{\perp} L_{\perp} \nabla_{\mu}\left[-\frac{1}{T} \ell_{\nu, \sigma}^{\mu \rho} \nabla_{\rho} u^{\sigma}\right] \\
h_{\alpha}^{v} \nabla_{\mu} T_{\nu}^{(\mathrm{q}) \mu} & =-h_{\alpha}^{v} c_{\|} L_{\|} \nabla_{\mu}\left[-\frac{1}{T} \ell_{\nu, \sigma}^{\mu \rho} \nabla_{\rho} u^{\sigma}\right] \\
\nabla_{\mu}\left(n u^{\mu}\right) & =\sqrt{h} \frac{\partial^{2} \tilde{s}}{\partial \tilde{n}^{2}} M \nabla_{\mu}\left[m^{\mu \nu} \partial_{\nu}\left(-\frac{\mu}{T}\right)\right]
\end{aligned}
$$

We see that general covariance requires that $c_{\perp} L_{\perp}=c_{\|} L_{\|}$ ( $\left.\equiv L_{\tilde{p}}\right)$. By assuming that $L_{\tilde{p}}$ and $L_{\tilde{n}} \equiv \sqrt{h}\left(-\partial^{2} \tilde{s} / \partial \tilde{n}^{2}\right) M$ can be regarded as being constant at this order of derivative expansion, the linear regression is expressed as current conservations:

$$
\nabla_{\mu} T_{v}^{\mu}=0, \quad \nabla_{\mu} n^{\mu}=0,
$$

where the energy-momentum tensor and the particle-number current are given by

$$
\begin{gathered}
T_{v}^{\mu} \equiv T_{v}^{(\mathrm{q}) \mu}+\tau_{v}^{(\mathrm{d}) \mu}=e u_{v} u^{\mu}+\tau_{\nu}^{(\mathrm{q}) \mu}+\tau_{\nu}^{(\mathrm{d}) \mu}, \\
n^{\mu} \equiv n u^{\mu}+v^{\mu},
\end{gathered}
$$

with

$$
\begin{aligned}
\tau_{\nu}^{(\mathrm{d}) \mu} & \equiv-\frac{1}{T} L_{\tilde{p}} \ell_{\nu, \sigma}^{\mu \rho} \nabla_{\rho} u^{\sigma}, \\
\nu^{\mu} & \equiv L_{\tilde{n}} m^{\mu \nu} \partial_{\nu}\left(-\frac{\mu}{T}\right) .
\end{aligned}
$$

For simple fluids that have no specific directions, the quantities given above can be parametrized as

$$
\begin{aligned}
\tau_{(\mathrm{q})}^{\mu \nu}= & P h^{\mu \nu} \quad(P: \text { pressure }), \\
\frac{1}{T} L_{\tilde{p}} \ell^{\mu \nu, \rho \sigma}= & \eta\left[h^{\mu \rho} h^{\nu \sigma}+h^{\mu \sigma} h^{\nu \rho}-(2 / D) h^{\mu \nu} h^{\rho \sigma}\right] \\
& +\zeta h^{\mu \nu} h^{\rho \sigma}, \\
L_{\tilde{n}} m^{\mu \nu}= & \sigma T^{2} h^{\mu \nu},
\end{aligned}
$$

where $\zeta, \eta$, and $\sigma(\geqslant 0)$ are, respectively, the bulk viscosity, the shear viscosity, and the diffusion constant. Note that the contributions from the rotation $h_{\mu}^{\rho} h_{v}^{\sigma} \nabla_{[\rho} u_{\sigma]}$ have been discarded since it vanishes in the assumption that the velocity field $u^{\mu}$ is hypersurface orthogonal. Then Eqs. (71)-(75) 
become the well-known conservation laws for viscous simple fluids in the Landau-Lifshitz frame:

$$
\begin{aligned}
\nabla_{\mu} T^{\mu \nu} & =0, \quad \nabla_{\mu} n^{\mu}=\nabla_{\mu}\left(n u^{\mu}+v^{\mu}\right)=0, \\
T^{\mu \nu} & \equiv e u^{\mu} u^{\nu}+\left(P-\zeta \nabla_{\rho} u^{\rho}\right) h^{\mu \nu}-2 \eta \nabla^{\langle\mu} u^{\nu\rangle}, \\
v^{\mu} & \equiv \sigma T^{2} h^{\mu \nu} \partial_{\nu}\left(-\frac{\mu}{T}\right) .
\end{aligned}
$$

Here and hereafter, we define $A^{\langle\mu \nu\rangle} \equiv(1 / 2) h_{\rho}^{\mu} h_{\sigma}^{\nu}\left(A^{\rho \sigma}+\right.$ $\left.A^{\sigma \rho}-(2 / D) h_{\alpha \beta} A^{\alpha \beta} h^{\rho \sigma}\right)$ for a tensor $A^{\mu \nu}$.

\section{B. More on the entropy production}

The entropy production rate can also be calculated in the following way once we know that the current conservations (79)-(81) hold. First we consider the increase of the entropy functional during the time interval $\Delta t\left(\gtrsim \epsilon_{\mathrm{s}}\right)$ :

$$
\begin{aligned}
\hat{S}(t & \left.+\Delta t ; \Sigma_{x}\left[L_{\mathrm{s}}\right]\right)-\hat{S}\left(t ; \Sigma_{x}\left[L_{\mathrm{s}}\right]\right)=\int_{t}^{t+\Delta t} d t \dot{\hat{S}}\left(t ; \Sigma_{x}\left[L_{\mathrm{s}}\right]\right) \\
= & \int_{t}^{t+\Delta t} d t \int_{\Sigma_{x}\left[L_{\mathrm{s}}\right]} d^{D} \boldsymbol{y} \dot{\tilde{s}}\left(\tilde{p}_{\mu}(t, \boldsymbol{y}), \tilde{n}(t, \boldsymbol{y}), g_{\mu \nu}(t, \boldsymbol{y})\right) \\
= & \int_{t}^{t+\Delta t} d t \int_{\Sigma_{x}\left[L_{\mathrm{s}}\right]} d^{D} \boldsymbol{y}\left[\frac{\partial \tilde{s}}{\partial \tilde{p}_{\mu}} \dot{\tilde{p}}_{\mu}+\frac{\partial \tilde{s}}{\partial \tilde{\tilde{n}}}+\frac{\partial \tilde{s}}{\partial g_{\mu \nu}} \dot{g}_{\mu \nu}\right] \\
= & \int_{t}^{t+\Delta t} \int_{\Sigma_{x}\left[L_{\mathrm{s}}\right]} d^{D+1} y \sqrt{-g}\left[-\frac{u^{\mu}}{T} \nabla_{\nu}\left(p_{\mu} u^{\nu}\right)\right. \\
& \left.+\left(-\frac{\mu}{T}\right) \nabla_{\mu}\left(n u^{\mu}\right)+\frac{1}{T} T_{(\mathrm{q})}^{\mu \nu} \nabla_{\nu} u_{\mu}\right] .
\end{aligned}
$$

Since $\nabla_{v} T^{\mu \nu}=0$ for $T^{\mu \nu}=p^{\mu} u^{v}+\tau^{\mu v} \equiv p^{\mu} u^{v}+\tau_{(\mathrm{q})}^{\mu \nu}+$ $\tau_{(\mathrm{d})}^{\mu \nu}, \quad \nabla_{\mu} n^{\mu}=0$ for $n^{\mu}=n u^{\mu}+v^{\mu}, \quad$ and $T_{(\mathrm{q})}^{\mu \nu} \nabla_{\nu} u_{\mu}=$ $\tau_{(\mathrm{q})}^{\mu \nu} \nabla_{\nu} u_{\mu}=-u_{\mu} \nabla_{\nu} \tau_{(\mathrm{q})}^{\mu \nu}$, the above equation can be rewritten as

$$
\begin{aligned}
\hat{S} & \left(t+\Delta t ; \Sigma_{x}\left[L_{\mathrm{s}}\right]\right)-\hat{S}\left(t ; \Sigma_{x}\left[L_{\mathrm{s}}\right]\right) \\
= & \int_{t}^{t+\Delta t} \int_{\Sigma_{x}\left[L_{\mathrm{s}}\right]} d^{D+1} y \sqrt{-g}\left[\frac{u^{\mu}}{T} \nabla_{\nu}\left(\tau_{\mu}^{\nu}-\tau_{\mu}^{(\mathrm{q}) \nu}\right)+\frac{\mu}{T} \nabla_{\mu} \nu^{\mu}\right] \\
= & \int_{t}^{t+\Delta t} \int_{\Sigma_{x}\left[L_{\mathrm{s}}\right]} d^{D+1} y \sqrt{-g}\left[\frac{u^{\mu}}{T} \nabla_{\nu} \tau_{\mu}^{(\mathrm{d}) \nu}+\frac{\mu}{T} \nabla_{\mu} \nu^{\mu}\right] .
\end{aligned}
$$

The first equality means that $\tau_{\mu}^{\nu}$ equals $\tau_{\mu}^{(\mathrm{q}) \nu}$ in the absence of dissipation, and thus shows that the equality $\tau_{\mu}^{(\mathrm{r}) v}=\tau_{\mu}^{(\mathrm{q}) \nu}$ holds for fluids. By integrating by parts, the equation can be further rewritten as

$$
\begin{aligned}
\hat{S}(t+ & \left.\Delta t ; \Sigma_{x}\left[L_{\mathrm{s}}\right]\right)-\hat{S}\left(t ; \Sigma_{x}\left[L_{\mathrm{s}}\right]\right) \\
= & \int_{t}^{t+\Delta t} \int_{\Sigma_{x}\left[L_{\mathrm{s}}\right]} d^{D+1} y \sqrt{-g} \\
& \times\left[-\frac{1}{T} \tau_{(\mathrm{d})}^{\mu v} K_{\mu \nu}+v^{\mu} \partial_{\mu}\left(-\frac{\mu}{T}\right)+\nabla_{\mu}\left(\frac{\mu}{T} v^{\mu}\right)\right] .
\end{aligned}
$$

The last term on the right-hand side is the effect from the surroundings so that the entropy production in the bulk is given by

$$
\begin{aligned}
& \hat{S}\left(t+\Delta t ; \Sigma_{x}\left[L_{\mathrm{s}}\right]\right)-\hat{S}\left(t ; \Sigma_{x}\left[L_{\mathrm{s}}\right]\right) \\
& -\int_{t}^{t+\Delta t} \int_{\Sigma_{x}\left[L_{\mathrm{s}}\right]} d^{D+1} y \sqrt{-g} \nabla_{\mu}\left(\frac{\mu}{T} v^{\mu}\right) \\
& =\int_{t}^{t+\Delta t} \int_{\Sigma_{x}\left[L_{\mathrm{s}}\right]} d^{D+1} y \sqrt{-g} \\
& \quad \times\left[-\frac{1}{T} \tau_{(\mathrm{d})}^{\mu v} K_{\mu \nu}+v^{\mu} \partial_{\mu}\left(-\frac{\mu}{T}\right)\right] .
\end{aligned}
$$

Since $\dot{\tilde{s}}$ can be expressed as $\sqrt{-g} \nabla_{\mu}\left(s u^{\mu}\right)$, the left-hand side of Eq. (85) can be written as $\int_{t}^{t+\Delta t} \int_{\Sigma_{x}\left[L_{\mathrm{s}}\right]} d^{D+1} y \sqrt{-g} \nabla_{\mu}\left[s u^{\mu}-\right.$ $\left.(\mu / T) \nu^{\mu}\right]$. Thus, by defining the entropy current as $s^{\mu} \equiv$ $s u^{\mu}-(\mu / T) v^{\mu}$, Eq. (85) can be expressed in a local form as

$$
\begin{aligned}
\nabla_{\mu} s^{\mu}= & -\frac{1}{T} \tau_{(\mathrm{d})}^{\mu \nu} K_{\mu \nu}+v^{\mu} \partial_{\mu}\left(-\frac{\mu}{T}\right) \\
= & \frac{\zeta}{T}\left(h^{\mu \nu} K_{\mu \nu}\right)^{2}+\frac{2 \eta}{T} K^{\langle\mu \nu\rangle} K_{\langle\mu \nu\rangle} \\
& +\sigma T^{2} h^{\mu \nu} \partial_{\mu}\left(-\frac{\mu}{T}\right) \partial_{\nu}\left(-\frac{\mu}{T}\right) .
\end{aligned}
$$

Equation (86) might seem to be inconsistent with the entropy production rate given in Eq. (42), because Eq. (42) predicts that the entropy production rate is the spatial integral of a quadratic form in $\nabla_{\mu} \nu^{\mu}$ and $\nabla_{\mu} \tau_{(\mathrm{d})}^{\mu \nu}$. However, they are actually consistent. In fact, suppose that we expand $u^{\mu} / T$ and $\mu / T$ in Eq. (83) around their equilibrium values $\left(u_{0}^{\mu} / T_{0}\right)\left(y ; L_{\mathrm{s}}\right)$ and $\left(\mu_{0} / T_{0}\right)\left(y ; L_{\mathrm{s}}\right)$ as

$$
\begin{aligned}
\frac{u^{\mu}}{T} & =\frac{u_{0}^{\mu}}{T_{0}}+\left(\frac{u^{\mu}}{T}-\frac{u_{0}^{\mu}}{T_{0}}\right), \\
\frac{\mu}{T} & =\frac{\mu_{0}}{T_{0}}+\left(\frac{\mu}{T}-\frac{\mu_{0}}{T_{0}}\right) .
\end{aligned}
$$

Since the deviations $u^{\mu} / T-u_{0}^{\mu} / T_{0}$ and $\mu / T-\mu_{0} / T_{0}$ are proportional to their thermodynamic forces, $\delta \Delta \hat{S} / \delta \tilde{p}_{\mu}$ and $\delta \Delta \hat{S} / \delta \tilde{n}$, respectively, they can be expressed in the following form:

$$
\begin{aligned}
\frac{u^{\mu}}{T}-\frac{u_{0}^{\mu}}{T_{0}} & =-\nabla_{\nu}\left[\gamma^{\mu \nu, \rho \sigma} \nabla_{\rho}\left(\frac{u_{\sigma}}{T}\right)\right], \\
\frac{\mu}{T}-\frac{\mu_{0}}{T_{0}} & =-\nabla_{\mu}\left[\gamma^{\mu \nu} \partial_{\nu}\left(\frac{\mu}{T}\right)\right],
\end{aligned}
$$

where $\gamma^{\mu \nu, \rho \sigma}$ and $\gamma^{\mu \nu}$ are some positive semidefinite matrices. Substituting them into Eq. (83), we have the following equation:

$$
\begin{aligned}
\dot{\hat{S}}(t ; & \left.\Sigma_{x}\left[L_{\mathrm{s}}\right]\right)-\int_{\Sigma_{x}\left[L_{\mathrm{s}}\right]} d^{D} \boldsymbol{y} \sqrt{-g} \nabla_{\nu}\left(\frac{u_{0}^{\mu}}{T_{0}} \tau_{\mu}^{(\mathrm{d}) \nu}+\frac{\mu_{0}}{T_{0}} v^{\nu}\right) \\
= & \int_{\Sigma_{x}\left[L_{\mathrm{s}}\right]} d^{D} \boldsymbol{y} \sqrt{-g}\left[-\nabla_{\nu}\left[\left(\gamma^{\mu \nu, \rho \sigma} / T\right) K_{\rho \sigma}\right] \nabla_{\lambda} \tau_{\mu}^{(\mathrm{d}) \lambda}\right. \\
& \left.\quad-\nabla_{\mu}\left[\gamma^{\mu \nu} \partial_{\nu}(\mu / T)\right] \nabla_{\rho} \nu^{\rho}\right] .
\end{aligned}
$$




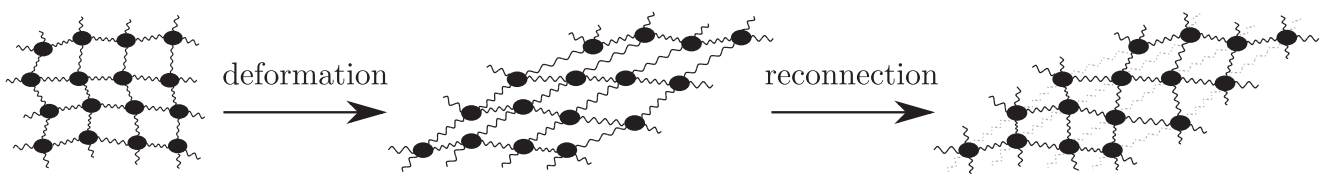

FIG. 4. Processes of deformation and strain relaxation.

Here $\left(u_{0}^{\mu} / T_{0}\right) \tau_{\mu}^{(\mathrm{d}) v}$ can be neglected at this order of approximation because $u_{0}^{\mu} \tau_{\mu}^{(\mathrm{d}) v} \simeq u^{\mu} \tau_{\mu}^{(\mathrm{d}) v}=0$, while $\nabla_{\nu}\left[\left(\mu_{0} / T_{0}\right) v^{\nu}\right]$ can be replaced by $\nabla_{\nu}\left[(\mu / T) \nu^{\nu}\right]$. Thus, the second term on the left-hand side of Eq. (91) can be written as $-\int_{\Sigma_{x}\left[L_{\mathrm{s}}\right]} d^{D} \boldsymbol{y} \sqrt{-g} \nabla_{\nu}\left[(\mu / T) v^{v}\right]$, so that the left-hand side of Eq. (91) agrees with that of Eq. (85). We thus find that the entropy production rate in the bulk can also be written as a quadratic form in $\nabla_{\mu} \nu^{\mu}$ and $\nabla_{\mu} \tau_{(\mathrm{d})}^{\mu \nu}$.

\section{RELATIVISTIC VISCOELASTIC FLUID MECHANICS}

\section{A. Intrinsic metric}

Once the motion of a material is specified by a velocity field $u^{\mu}(x)$, the shape of the time slice normal to $u^{\mu}$ is represented by the induced metric (4), $h_{\mu \nu}=g_{\mu \nu}+u_{\mu} u_{\nu}$. If the relaxation time for the deformation of shape can be regarded as being very short, then, as discussed in Sec. III, the fundamental relation, $\tilde{s}(x)=\tilde{\sigma}\left(\tilde{e}(x), \tilde{n}(x), g_{\mu \nu}(x)\right)$, does not depend on the hysteresis of the shape $h_{\mu \nu}(x)$. Almost all materials (besides fluids), however, do not have such short relaxation times for the deformation of shape, and the time evolution of shape may not be described as a Markovian process; all the preceding history needs to be known in order to predict the future behavior of the system at a given initial time slice. One then needs to extend the formalism such that the equations contain time derivatives of higher orders. This is out of the scope of the standard nonequilibrium thermodynamics.

However, a class of materials still allow a standard thermodynamic description even when their relaxation time of shape is not short. This is performed by replacing the introduction of higher time derivatives with that of extra dynamical degrees of freedom. Viscoelastic materials considered below belong to this class of materials.
According to the definition of Maxwell, viscoelastic materials behave as elastic solids at short time scales and as viscous fluids at long time scales (see, e.g., Sec. 36 in [14]). In order to understand how such materials evolve in time, we consider a material consisting of many molecules bonding each other and assume that the molecules first stay at their equilibrium positions in the absence of strains (as in the leftmost illustration of Fig. 4) [11,12]. We now suppose that an external force is applied to deform the material. An internal strain is then produced in the body, and according to the definition, the accompanied internal stress can be treated as an elastic force, at least during short intervals of time. However, if we keep the deformation much longer than the relaxation time (characteristic to each material), then the bonding structure changes to maximize the entropy, and the internal strain vanishes eventually as in the rightmost of Fig. 4 . The point is that two figures (the central and the rightmost) have the same shape (same induced metric) $h_{\mu \nu}$, but different bonding structures.

In order to describe the internal bonding structure, we introduce at each moment another dynamical variable to be called the intrinsic metric, which measures the shape that the material would take when all the internal strains are removed virtually [10-12]. To define such states, around each space-time point $x$, we consider a small spatial subregion whose linear size $\lambda$ is much smaller than $L_{\mathrm{s}}$ and enclose the subregion with an adiabatic and impermeable wall of vanishing tension (see Fig. 5). We then cut it out of the bulk $\Sigma_{x}\left[L_{\mathrm{s}}\right]$ and leave it for a long time (comparable to $L_{\mathrm{t}}$ ). The subregion will then undergo a relaxation to reach its equilibrium state. This causes a diffeomorphism in $(D+1)$-dimensional spacetime from the subregion to another subregion, mapping the original (or real) positions of material particles to their new positions in the virtual equilibrium, $f_{\lambda}: x \mapsto f_{\lambda}(x)$. Since the absolute value of the extrinsic curvature gets reduced in the course of relaxation, this diffeomorphism does not necessarily

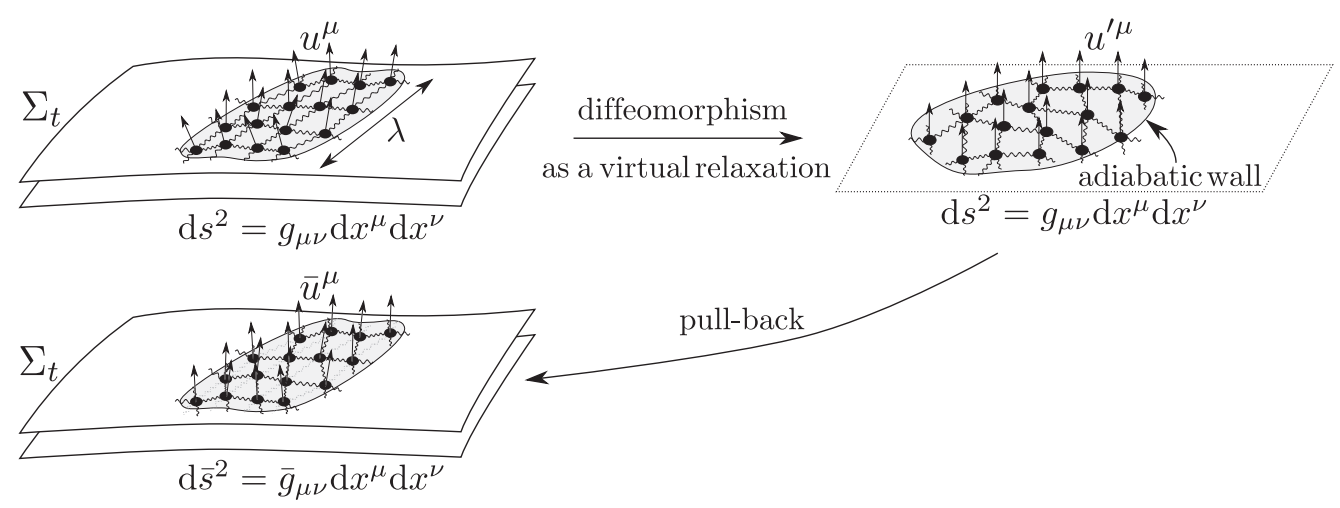

FIG. 5. The procedure to obtain the intrinsic metric. 
preserve the foliation, and the velocity field $u_{\mu}(x)$ will change as $u_{\mu}(x) \rightarrow u_{\mu}^{\prime}(x) .{ }^{5}$ We then consider the pullbacks of the resulting fields in the virtual equilibrium:

$$
\bar{u}_{\mu}(x ; \lambda) \equiv\left(f_{\lambda}^{*} u^{\prime}\right)_{\mu}(x), \quad \bar{g}_{\mu \nu}(x ; \lambda) \equiv\left(f_{\lambda}^{*} g\right)_{\mu \nu}(x),
$$

and define the intrinsic velocity field $\bar{u}_{\mu}(x)$ and the intrinsic metric $\bar{g}_{\mu \nu}(x)$ as the values in the limit $\lambda \rightarrow 0$ :

$$
\bar{u}_{\mu}(x) \equiv \lim _{\lambda \rightarrow 0}\left(f_{\lambda}^{*} u^{\prime}\right)_{\mu}(x), \quad \bar{g}_{\mu \nu}(x) \equiv \lim _{\lambda \rightarrow 0}\left(f_{\lambda}^{*} g\right)_{\mu \nu}(x) .
$$

Since $\bar{g}^{\mu \nu} \bar{u}_{v}$ is no longer orthogonal to the original hypersurface, the intrinsic metric $\bar{g}_{\mu \nu}(x)$ may have tilted components. We parametrize $\bar{g}_{\mu \nu}$ as

$$
\begin{aligned}
\bar{g}_{\mu \nu} & =-(1+2 \theta) u_{\mu} u_{\nu}-\varepsilon_{\mu} u_{\nu}-\varepsilon_{\nu} u_{\mu}+\bar{h}_{\mu \nu} \\
\left(\varepsilon_{\mu} u^{\mu}\right. & \left.=0, \quad h_{\mu \nu} u^{\nu}=0, \quad \bar{h}_{\mu \nu} u^{\nu}=0\right) .
\end{aligned}
$$

The quantity $\sqrt{1+2 \theta}$ represents the ratio of the temperature in the presence of strains to that in the absence of strains, because the Tolman law $(\mathrm{T}=N T=\bar{N} \bar{T})$ tells that $T / \bar{T}=$ $\bar{N} / N=\sqrt{1+2 \theta}$, that is, $\theta=\left(T^{2}-\bar{T}^{2}\right) / 2 \bar{T}^{2} \simeq(T-\bar{T}) / \bar{T}$. The quantity $\varepsilon_{\mu}$ represents the relative velocity of the real path of a material particle to that of its virtual path. The spatial metric $\bar{h}_{\mu \nu}$ is the same with the "strain metric" introduced by Eckart to embody "the principle of relaxability-in-the-small" in anelasticity [10]. (This was reinvented in [11] in the light of the covariant description of viscoelasticity under the foliation preserving diffeomorphisms.) We further introduce the strain

\footnotetext{
${ }^{5}$ This virtual process will also cause a change in the energy distribution, and accordingly, the background metric may change as to satisfy the Einstein equation. However, such change can be neglected here because this is a higher-order effect in our derivative expansion.
}

tensor

$$
\begin{aligned}
E_{\mu \nu}(x) & \equiv \frac{1}{2}\left[g_{\mu \nu}(x)-\bar{g}_{\mu \nu}(x)\right] \\
& =\theta u_{\mu} u_{\nu}+\frac{1}{2}\left(\varepsilon_{\mu} u_{\nu}+\varepsilon_{\nu} u_{\mu}\right)+\varepsilon_{\mu \nu},
\end{aligned}
$$

where

$$
\varepsilon_{\mu \nu}(x) \equiv \frac{1}{2}\left[h_{\mu \nu}(x)-\bar{h}_{\mu \nu}(x)\right]
$$

is the spatial strain tensor. The explicit forms of $h_{\mu \nu}$ and $\bar{h}_{\mu \nu}$ under various deformations can be found in [12]. Note that if we define the extrinsic curvature associated with the spatial intrinsic metric $\bar{h}_{\mu \nu}$ as

$$
\bar{K}_{\mu \nu} \equiv \frac{1}{2} £_{u} \bar{h}_{\mu \nu}=\frac{1}{2}\left(u^{\lambda} \partial_{\lambda} \bar{h}_{\mu \nu}+\partial_{\mu} u^{\lambda} \bar{h}_{\lambda \mu}+\partial_{\nu} u^{\lambda} \bar{h}_{\mu \lambda}\right),
$$

the following identity holds:

$$
£_{u} \varepsilon_{\mu \nu}=K_{\mu \nu}-\bar{K}_{\mu \nu} .
$$

We denote the contraction of a spatial tensor $A_{\mu \nu}$ with $g^{\mu \nu}$ by tr $A$ so that

$$
\begin{aligned}
\operatorname{tr} \varepsilon & \equiv g^{\mu \nu} \varepsilon_{\mu \nu}=h^{\mu \nu} \varepsilon_{\mu \nu}, \\
\operatorname{tr} K & \equiv g^{\mu \nu} K_{\mu \nu}=h^{\mu \nu} K_{\mu \nu} .
\end{aligned}
$$

\section{B. Linear regression and the rheology equations}

We now apply the framework of Sec. II with the following identifications:

\begin{tabular}{cccccc}
$b^{A}$ & $b_{0}^{A}$ & $\tilde{c}^{I}$ & $\beta_{I}$ & $d^{P}$ & $\left(\partial \tilde{s} / \partial d^{P}\right)$ \\
\hline$\varepsilon_{\mu \nu}$ & 0 & $\tilde{p}_{\mu}$ & $-u^{\mu} / T$ & $g_{\mu \nu}$ & $\sqrt{h} T_{(\mathrm{q})}^{\mu \nu} / 2 T$ \\
$\varepsilon_{\mu}$ & 0 & $\tilde{n}$ & $-\mu / T$ & & \\
$\theta$ & 0 & & & &
\end{tabular}

Following the argument used in deriving Eq. (50), the entropy functional (31) are generically expanded as follows:

$$
\begin{aligned}
& \Delta \hat{S}\left(t ; \Sigma_{x}\left[L_{\mathrm{s}}\right]\right)=-\frac{1}{2} \int_{\Sigma_{x}\left[L_{\mathrm{s}}\right]} d^{D} \boldsymbol{y} \sqrt{-g}\left[\left(\begin{array}{cc}
\varepsilon_{\langle\mu \nu\rangle} & \nabla^{\langle\mu}\left(\partial \tilde{s} / \partial \tilde{p}_{\nu\rangle}\right)
\end{array}\right)\left(\begin{array}{cc}
\ell_{1}^{\langle\mu \nu\rangle,\langle\rho \sigma\rangle} & \ell_{2\langle\rho \sigma\rangle}^{\langle\mu \nu\rangle,} \\
\ell_{2\langle\mu \nu\rangle,}^{\langle\rho \sigma\rangle} & \ell_{3\langle\mu \nu\rangle,\langle\rho \sigma\rangle}
\end{array}\right)\left(\begin{array}{c}
\varepsilon_{\langle\rho \sigma\rangle} \\
\nabla^{\langle\rho}\left(\partial \tilde{s} / \partial \tilde{p}_{\sigma\rangle}\right)
\end{array}\right)\right. \\
& \left.+\left(\begin{array}{ll}
\varepsilon_{\mu} & \partial_{\mu}(\partial \tilde{s} / \partial \tilde{n})
\end{array}\right)\left(\begin{array}{cc}
\ell_{1}^{\mu \nu} & \ell_{2}^{\mu \nu} \\
\ell_{2}^{\mu \nu} & \ell_{3}^{\mu \nu}
\end{array}\right)\left(\begin{array}{c}
\varepsilon_{v} \\
\partial_{\nu}(\partial \tilde{s} / \partial \tilde{n})
\end{array}\right)+\left(\begin{array}{lll}
\operatorname{tr} \varepsilon & \theta & \nabla_{\mu}\left(\partial \tilde{s} / \partial \tilde{p}_{\mu}\right)
\end{array}\right)\left(\begin{array}{ccc}
\ell_{1}^{\mathrm{s}} & \ell_{2}^{\mathrm{s}} & \ell_{4}^{\mathrm{s}} \\
\ell_{2}^{\mathrm{s}} & \ell_{3}^{\mathrm{s}} & \ell_{5}^{\mathrm{s}} \\
\ell_{4}^{\mathrm{s}} & \ell_{5}^{\mathrm{s}} & \ell_{6}^{\mathrm{s}}
\end{array}\right)\left(\begin{array}{c}
\operatorname{tr} \varepsilon \\
\theta \\
\nabla_{\mu}\left(\partial \tilde{s} / \partial \tilde{p}_{\mu}\right)
\end{array}\right)\right]
\end{aligned}
$$

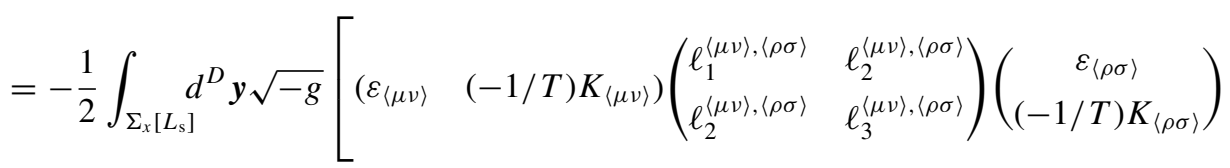

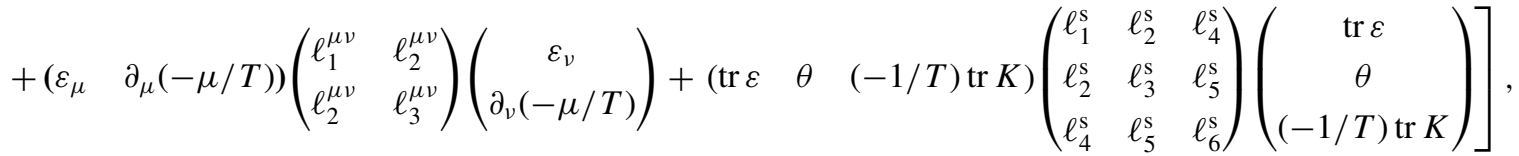


where the coefficient matrices are symmetric and positive semidefinite, and their indices are taken to be all orthogonal to $u^{\mu}$. Note that we again have used the fact that the rotation $h_{\mu}^{\rho} h_{v}^{\sigma} \nabla_{[\rho} u_{\sigma]}$ vanishes here. Since the matrices must be invariant tensors, we can assume that they take the following form:

$$
\begin{aligned}
\left(\begin{array}{ll}
\ell_{1}^{\langle\mu \nu\rangle,\langle\rho \sigma\rangle} & \ell_{2}^{\langle\mu \nu\rangle,\langle\rho \sigma\rangle} \\
\ell_{2}^{\langle\mu \nu\rangle,\langle\rho \sigma\rangle} & \ell_{3}^{\langle\mu \nu\rangle,\langle\rho \sigma\rangle}
\end{array}\right) & =\left(\begin{array}{ll}
\ell_{1}^{\mathrm{t}} & \ell_{2}^{\mathrm{t}} \\
\ell_{2}^{\mathrm{t}} & \ell_{3}^{\mathrm{t}}
\end{array}\right) h_{\mu^{\prime}}^{\langle\mu} h_{\nu^{\prime}}^{\nu\rangle} h^{\mu^{\prime} \rho} h^{\nu^{\prime} \sigma}, \\
\left(\begin{array}{ll}
\ell_{1}^{\mu \nu} & \ell_{2}^{\mu \nu} \\
\ell_{2}^{\mu \nu} & \ell_{3}^{\mu \nu}
\end{array}\right) & =\left(\begin{array}{ll}
\ell_{1}^{\mathrm{v}} & \ell_{2}^{\mathrm{v}} \\
\ell_{2}^{\mathrm{v}} & \ell_{3}^{\mathrm{v}}
\end{array}\right) h^{\mu \nu},
\end{aligned}
$$

where $\left(\begin{array}{ll}\ell_{1}^{\mathrm{t}} & \ell_{2}^{\mathrm{t}} \\ \ell_{2}^{\mathrm{t}} & \ell_{3}^{\mathrm{t}}\end{array}\right)$ and $\left(\begin{array}{ll}\ell_{1}^{\mathrm{v}} & \ell_{2}^{\mathrm{v}} \\ \ell_{2}^{\mathrm{v}} & \ell_{3}^{\mathrm{v}}\end{array}\right)$ are positive semidefinite. Note that $\ell_{k}^{\langle\mu \nu\rangle,\langle\rho \sigma\rangle} \varepsilon_{\langle\rho \sigma\rangle}=\ell_{k}^{\mathrm{t}} \varepsilon^{\langle\mu \nu\rangle} \quad(k=1,2,3)$. The functional derivatives are then evaluated to be

$$
\begin{aligned}
\frac{\delta \Delta \hat{S}}{\delta \varepsilon_{\langle\mu \nu\rangle}}= & -\sqrt{-g}\left[\ell_{1}^{\mathrm{t}} \varepsilon^{\langle\mu \nu\rangle}-\left(\ell_{2}^{\mathrm{t}} / T\right) K^{\langle\mu \nu\rangle}\right], \\
\frac{\delta \Delta \hat{S}}{\delta \varepsilon_{\mu}}= & -\sqrt{-g} h^{\mu \nu}\left[\ell_{1}^{\mathrm{v}} \varepsilon_{v}+\ell_{2}^{\mathrm{v}} \partial_{\nu}(-\mu / T)\right]\left(=\left[\frac{\delta \Delta \hat{S}}{\delta \varepsilon_{\mu}}\right]_{\|}\right), \\
\frac{\delta \Delta \hat{S}}{\delta(\operatorname{tr} \varepsilon)}= & -\sqrt{-g}\left[\ell_{1}^{\mathrm{s}} \operatorname{tr} \varepsilon+\ell_{2}^{\mathrm{s}} \theta-\left(\ell_{4}^{\mathrm{s}} / T\right) \operatorname{tr} K\right], \\
\frac{\delta \Delta \hat{S}}{\delta \theta}= & -\sqrt{-g}\left[\ell_{2}^{\mathrm{s}} \operatorname{tr} \varepsilon+\ell_{3}^{\mathrm{s}} \theta-\left(\ell_{5}^{\mathrm{s}} / T\right) \operatorname{tr} K\right], \\
\frac{\delta \Delta \hat{S}}{\delta \tilde{p}_{\mu}}= & -N\left(c_{\perp} u^{\mu} u^{\nu}+c_{\|} h^{\mu \nu}\right) \nabla^{\rho}\left[\ell_{2}^{\mathrm{t}} \varepsilon_{\langle\nu \rho\rangle}-\left(\ell_{3}^{\mathrm{t}} / T\right) K_{\langle v \rho\rangle}\right. \\
& \left.+\left(\ell_{4}^{\mathrm{s}} \operatorname{tr} \varepsilon+\ell_{5}^{\mathrm{s}} \theta-\left(\ell_{6}^{\mathrm{s}} / T\right) \operatorname{tr} K\right) h_{\nu \rho}\right] \\
= & {\left[\frac{\delta \Delta \hat{S}}{\delta \tilde{p}_{\mu}}\right]_{\perp}+\left[\frac{\delta \Delta \hat{S}}{\delta \tilde{p}_{\mu}}\right]_{\|}, } \\
\frac{\delta \Delta \hat{S}}{\delta \tilde{n}}= & -\sqrt{-g}\left(-\frac{\partial^{2} \tilde{s}}{\partial \tilde{n}^{2}}\right) \nabla_{\mu}\left[\ell_{2}^{\mathrm{v}} h^{\mu \nu} \varepsilon_{\nu}+\ell_{3}^{\mathrm{v}} h^{\mu \nu} \partial_{\nu}(-\mu / T)\right],
\end{aligned}
$$

where we have made the following parametrization as in Eq. (55):

$$
\left.\sqrt{h} \frac{\partial^{2} \tilde{s}}{\partial \tilde{p}_{\mu} \partial \tilde{p}_{\nu}}\right|_{0}=-c_{\perp} u^{\mu} u^{\nu}-c_{\|} h^{\mu \nu} .
$$

Note that $\delta \Delta \hat{S} / \delta \varepsilon_{\mu}$ has only the components tangent to the time slices. form:

The equations of linear regression are given in the following

$$
\begin{aligned}
{\left[\dot{\varepsilon}_{\langle\mu \nu\rangle}\right]_{\mathrm{irr}} } & \equiv \frac{1}{\sqrt{h}} L^{\varepsilon_{\langle\mu \nu\rangle} \varepsilon_{\langle\rho \sigma\rangle}} \frac{\delta \Delta \hat{S}}{\delta \varepsilon_{\rho \sigma}}, \\
{\left[\dot{\varepsilon}_{\mu}\right]_{\mathrm{irr} \perp} } & \equiv \frac{1}{\sqrt{h}} L_{\perp}^{\varepsilon_{\mu} \varepsilon_{\nu}}\left[\frac{\delta \Delta \hat{S}}{\delta \varepsilon_{\nu}}\right]_{\perp} \equiv 0, \\
{\left[\dot{\varepsilon}_{\mu}\right]_{\mathrm{irr} \|} } & \equiv \frac{1}{\sqrt{h}} L_{\|}^{\varepsilon_{\mu} \varepsilon_{\nu}}\left[\frac{\delta \Delta \hat{S}}{\delta \varepsilon_{v}}\right]_{\|}, \\
\left(\begin{array}{cc}
{\left[(\operatorname{tr} \varepsilon)^{\cdot}\right]_{\operatorname{irr}}} \\
{[\dot{\theta}]_{\text {irr }}}
\end{array}\right) & \equiv \frac{1}{\sqrt{h}}\left(\begin{array}{ll}
L^{\operatorname{tr} \varepsilon \operatorname{tr} \varepsilon} & L^{\operatorname{tr} \varepsilon \theta} \\
L^{\operatorname{tr} \varepsilon \theta} & L^{\theta \theta}
\end{array}\right)\left(\begin{array}{c}
\frac{\delta \Delta \hat{S}}{\delta(\operatorname{tr} \varepsilon)} \\
\frac{\delta \Delta \hat{S}}{\delta \theta}
\end{array}\right),
\end{aligned}
$$

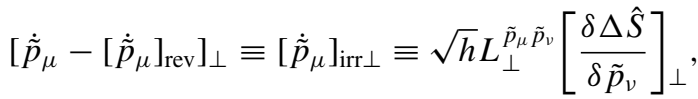

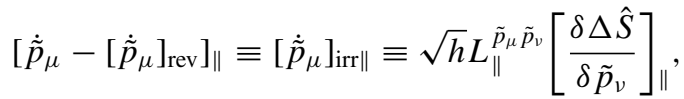

$$
\begin{aligned}
& \dot{\tilde{n}}-[\dot{\tilde{n}}]_{\mathrm{rev}} \equiv[\dot{\tilde{n}}]_{\mathrm{irr}} \equiv \sqrt{h} L^{\tilde{n} \tilde{n}} \frac{\delta \Delta \hat{S}}{\delta \tilde{n}} .
\end{aligned}
$$

We here do not consider the direct coupling between the variables $\varepsilon_{\mu}$ and $\tilde{p}_{\mu}$, expecting that it is negligible. Note also that $\operatorname{tr} \dot{\varepsilon} \equiv g^{\mu \nu} \dot{\varepsilon}_{\mu \nu}=h^{\mu \nu} \dot{\varepsilon}_{\mu \nu}$ can be replaced by $(\operatorname{tr} \varepsilon)^{\cdot}$ at this order of approximation because $(\operatorname{tr} \varepsilon)^{\cdot}-\operatorname{tr} \dot{\varepsilon}=\left(h^{\mu \nu}\right)^{\circ} \varepsilon_{\mu \nu}=$ $-2 N K^{\mu v} \varepsilon_{\mu \nu}$. As we have done for fluids, we make the following irreducible decompositions under the group $\mathrm{O}(D)$ in a local inertial frame:

$$
\begin{aligned}
L^{\varepsilon_{\langle\mu \nu\rangle} \varepsilon_{\langle\rho \sigma\rangle}} & \equiv L^{\mathrm{t}} h_{\langle\mu}^{\mu^{\prime}} h_{\nu\rangle}^{v^{\prime}} h_{\mu^{\prime} \rho} h_{\nu^{\prime} \sigma}, \\
L_{\|}^{\varepsilon_{\mu} \varepsilon_{\nu}} & \equiv L^{\mathrm{v}} h_{\mu \nu}, \\
L^{\operatorname{tr} \varepsilon \operatorname{tr} \varepsilon} & \equiv L_{1}^{\mathrm{s}}, \\
L^{\operatorname{tr} \varepsilon \theta} & \equiv L_{2}^{\mathrm{s}}, \\
L^{\theta \theta} & \equiv L_{3}^{\mathrm{s}}, \\
L_{\perp}^{\tilde{p}_{\mu} \tilde{p}_{\nu}} & \equiv L_{\perp} u_{\mu} u_{\nu}, \\
L_{\|}^{\tilde{p}_{\mu} \tilde{p}_{v}} & \equiv L_{\|} h_{\mu \nu}, \\
L^{\tilde{n} \tilde{n}} & \equiv M .
\end{aligned}
$$

Thus, by assuming that the reversible evolution of $\tilde{p}_{\mu}$ and $\tilde{n}$ is expressed with reversible currents $\tau_{\mu}^{(\mathrm{r}) \nu}$ and $\nu_{(\mathrm{r})}^{\mu}$ (whose explicit form will be given later for a simple case) as

$$
\begin{gathered}
{[\dot{\tilde{\tilde{p}}}]_{\mathrm{rev}}=-\sqrt{-g} \nabla_{\nu} \tau_{\mu}^{(\mathrm{r}) v},} \\
{[\dot{\tilde{n}}]_{\mathrm{rev}}=-\sqrt{-g} \nabla_{\mu} \nu_{(\mathrm{r})}^{\mu},}
\end{gathered}
$$

Eqs. (115)-(117) become

$$
\begin{gathered}
{\left[\nabla_{\mu}\left(p^{v} u^{\mu}+\tau_{(\mathrm{r})}^{\mu \nu}\right)\right]_{\perp}} \\
=-c_{\perp} L_{\perp}\left(-u^{v} u_{\lambda}\right) \nabla_{\mu}\left[\ell_{2}^{\mathrm{t}} \varepsilon^{\langle\mu \lambda\rangle}-(1 / T) \ell_{3}^{\mathrm{t}} K^{\langle\mu \lambda\rangle}\right. \\
\left.\quad+\left(\ell_{4}^{\mathrm{s}} \operatorname{tr} \varepsilon+\ell_{5}^{\mathrm{s}} \theta-(1 / T) \ell_{6}^{\mathrm{s}} \operatorname{tr} K\right) h^{\mu \lambda}\right] \\
{\left[\nabla_{\mu}\left(p^{v} u^{\mu}+\tau_{(\mathrm{r})}^{\mu \nu}\right)\right]_{\|}} \\
=-c_{\|} L_{\|} h_{\lambda}^{v} \nabla_{\mu}\left[\ell_{2}^{\mathrm{t}} \varepsilon^{\langle\mu \lambda\rangle}-(1 / T) \ell_{3}^{\mathrm{t}} K^{\langle\mu \lambda\rangle}\right. \\
\left.\quad+\left(\ell_{4}^{\mathrm{s}} \operatorname{tr} \varepsilon+\ell_{5}^{\mathrm{s}} \theta-(1 / T) \ell_{6}^{\mathrm{s}} \operatorname{tr} K\right) h^{\mu \lambda}\right] \\
\nabla_{\mu}\left(n u^{\mu}+v_{(\mathrm{r})}^{\mu}\right) \\
=-\sqrt{h}\left(-\frac{\partial^{2} \tilde{s}}{\partial \tilde{n}^{2}}\right) M \nabla_{\mu}\left[\ell_{2}^{\mathrm{v}} h^{\mu v} \varepsilon_{v}+\ell_{3}^{\mathrm{v}} h^{\mu \nu} \partial_{v}(-\mu / T)\right]
\end{gathered}
$$

Assuming again that $L_{\tilde{p}} \equiv c_{\perp} L_{\perp}=c_{\|} L_{\|} \quad$ and $\quad L_{\tilde{n}} \equiv$ $\sqrt{h}\left(-\partial^{2} \tilde{s} / \partial \tilde{n}^{2}\right) M$ are constant, the above equations can be written in the form of current conservations:

$$
\nabla_{\mu} T^{\mu v}=0, \quad \nabla_{\mu} n^{\mu}=0,
$$

where the conserved currents are given by

$$
\begin{aligned}
T^{\mu v} & \equiv e u^{\mu} u^{v}+\tau_{(\mathrm{r})}^{\mu \nu}+\tau_{(\mathrm{d})}^{\mu v}, \\
n^{\mu} & \equiv n u^{\mu}+v_{(\mathrm{r})}^{\mu}+v_{(\mathrm{d})}^{\mu},
\end{aligned}
$$


with the dissipative currents

$$
\begin{aligned}
\tau_{(\mathrm{d})}^{\mu \nu} \equiv & L_{\tilde{p}} \ell_{2}^{\mathrm{t}} \varepsilon^{\langle\mu \nu\rangle}-(1 / T) L_{\tilde{p}} \ell_{3}^{\mathrm{t}} K^{\langle\mu \nu\rangle} \\
& +L_{\tilde{p}}\left[\ell_{4}^{\mathrm{s}} \operatorname{tr} \varepsilon+\ell_{5}^{\mathrm{s}} \theta-(1 / T) \ell_{6}^{\mathrm{s}} \operatorname{tr} K\right] h^{\mu \nu}, \\
v_{(\mathrm{d})}^{\mu} \equiv & L_{\tilde{n}}\left[\ell_{2}^{\mathrm{v}} h^{\mu \nu} \varepsilon_{\nu}+\ell_{3}^{\mathrm{v}} h^{\mu \nu} \partial_{\nu}(-\mu / T)\right] .
\end{aligned}
$$

As for the $b^{A}$-type variables, the equations of linear regression Eqs. (111)-(114) can be represented in the following form:

$$
\begin{aligned}
£_{u} \varepsilon_{\langle\mu \nu\rangle}= & N^{-1}\left[\dot{\varepsilon}_{\langle\mu \nu\rangle}\right]_{\mathrm{rev}}-L_{\mathrm{t}} \ell_{1}^{\mathrm{t}} \varepsilon_{\langle\mu \nu\rangle}+\left(L_{\mathrm{t}} \ell_{2}^{\mathrm{t}} / T\right) K_{\langle\mu \nu\rangle}, \\
\left(£_{u} \varepsilon_{\mu}\right)_{\perp}= & N^{-1}\left[\dot{\varepsilon}_{\mu}\right]_{\mathrm{rev} \perp}, \\
\left(£_{u} \varepsilon_{\mu}\right)_{\|}= & N^{-1}\left[\dot{\varepsilon}_{\mu}\right]_{\mathrm{rev} \|}-L^{\mathrm{v}} h_{\mu}^{v}\left[\ell_{1}^{\mathrm{v}} \varepsilon_{v}+\ell_{2}^{\mathrm{v}} \partial_{\nu}(-\mu / T)\right], \\
£_{u}(\operatorname{tr} \varepsilon)= & N^{-1}\left[(\operatorname{tr} \varepsilon)^{\cdot}\right]_{\mathrm{rev}}-\left(L_{1}^{\mathrm{s}} \ell_{1}^{\mathrm{s}}+L_{2}^{\mathrm{s}} \ell_{2}^{\mathrm{s}}\right) \operatorname{tr} \varepsilon \\
& -\left(L_{1}^{\mathrm{s}} \ell_{2}^{\mathrm{s}}+L_{2}^{\mathrm{s}} \ell_{3}^{\mathrm{s}}\right) \theta+\left(L_{1}^{\mathrm{s}} \ell_{4}^{\mathrm{s}}+L_{2}^{\mathrm{s}} \ell_{5}^{\mathrm{s}}\right) \frac{1}{T} \operatorname{tr} K,
\end{aligned}
$$

$$
\begin{aligned}
£_{u} \theta= & N^{-1}[\dot{\theta}]_{\mathrm{rev}}-\left(L_{2}^{\mathrm{s}} \ell_{1}^{\mathrm{s}}+L_{3}^{\mathrm{s}} \ell_{2}^{\mathrm{s}}\right) \operatorname{tr} \varepsilon-\left(L_{2}^{\mathrm{s}} \ell_{2}^{\mathrm{s}}+L_{3}^{\mathrm{s}} \ell_{3}^{\mathrm{s}}\right) \theta \\
& +\left(L_{2}^{\mathrm{s}} \ell_{4}^{\mathrm{s}}+L_{3}^{\mathrm{s}} \ell_{5}^{\mathrm{s}}\right) \frac{1}{T} \operatorname{tr} K .
\end{aligned}
$$

They give the generally covariant extension of the rheology equations introduced in [10,11], and describe the dynamics of plastic deformations.

The current conservations (131)-(135) and the rheology equations (136)-(140) totally determine the time evolution of the variables $p_{\mu}, n, \varepsilon_{\mu \nu}, \varepsilon_{\mu}$, and $\theta$, and thus constitute the set of the fundamental equations that govern the dynamics of relativistic viscoelastic materials. Further studies of relativistic viscoelastic materials are performed in our subsequent paper [15].

We finally make a comment that the explicit forms of $\left[\dot{\varepsilon}_{\langle\mu \nu\rangle}\right]_{\mathrm{rev}},\left[\dot{\varepsilon}_{\mu}\right]_{\mathrm{rev}},\left[(\operatorname{tr} \varepsilon)^{\cdot}\right]_{\mathrm{rev}}$, and $[\dot{\theta}]_{\mathrm{rev}}$ depend on the system under consideration. For example, for the case when the reversible, isentropic evolution describes that of elastic materials, they are given by

$$
\begin{gathered}
\tau_{(\mathrm{r})}^{\mu \nu}=-2 \mathcal{G} \varepsilon^{\langle\mu \nu\rangle}-\mathcal{K}(\operatorname{tr} \varepsilon-a \theta) h^{\mu \nu}, \quad v_{(\mathrm{r})}^{\mu}=0, \\
N^{-1}\left[\dot{\varepsilon}_{\langle\mu \nu\rangle}\right]_{\mathrm{rev}}=K_{\langle\mu \nu\rangle}, \quad N^{-1}\left[(\operatorname{tr} \varepsilon)^{\cdot}\right]_{\mathrm{rev}}=\operatorname{tr} K, \\
{\left[\dot{\varepsilon}_{\mu}\right]_{\mathrm{rev}}=[\dot{\theta}]_{\mathrm{rev}}=0 .}
\end{gathered}
$$

Here the non-negative constants $\mathcal{G}$ and $\mathcal{K}$ represent the shear and bulk modulus, respectively, and the constant $a$ is proportional to the coefficient of thermal expansion. Equations (142) and (143) show that the intrinsic metric $\bar{h}_{\mu \nu}$ does not vary for reversible processes $\left(\left[\dot{\bar{h}}_{\mu \nu}\right]_{\mathrm{rev}}=\left[2 N \bar{K}_{\mu \nu}\right]_{\mathrm{rev}}=2 N K_{\mu \nu}-\right.$ $\left.\left[\dot{\varepsilon}_{\mu \nu}\right]_{\mathrm{rev}}=0\right)$. A few other examples of isentropic evolutions are also given in [15].

\section{CONCLUSION AND DISCUSSIONS}

In this paper, we have developed an entropic formulation of relativistic continuum mechanics, which includes the standard relativistic fluid theory. The discussion is based on the linear nonequilibrium thermodynamics, and we have proposed a local functional $\hat{S}$ which represents the total entropy of nonequilibrium states and is to be maximized in the course of evolution. We have applied this framework to constructing a relativistic theory of viscoelastic materials. As is intensively studied in our subsequent paper [15], this theory can deal with a wide class of continuum materials, including as special cases elastic materials, Maxwell materials, Kelvin-Voigt materials, relativistic viscous fluids, and the so-called simplified IsraelStewart fluids, and thus is expected to be the most universal description of single-component (not necessarily relativistic) continuum materials.

Our entropy functional $\hat{S}$ is a local functional. Thus, once the coefficients $\ell^{A B}, \ldots, \ell^{\mu I, v J}$ in Eq. (31) are determined for a given material, one can explicitly evaluate the difference of the entropy of any configuration from that in the global equilibrium, $\Delta \hat{S}=\hat{S}-\hat{S}_{0}$, as long as the configuration is not far from the global equilibrium; one only needs to measure the local values of thermodynamic variables (such as the values of temperature at each point) and put the obtained data into $\Delta \hat{S}$.

The next step would be to extend the current formalism as one can deal with more complicated systems like multicomponent viscoelastic materials or systems with extra variables like liquid crystals. Such extension is actually straightforward and is under current investigation.

Another interesting direction would be to extract from our analysis the information on the holography of gravitation. In fact, one would need to assign local entropy to the metric tensor when the system is analyzed in generic frames (other than the Landau-Lifshitz frame) and when material particles have nonvanishing accelerations [16]. If this point is carefully investigated within the framework of the Bogoliubov-BornGreen-Kirkwood-Yvon hierarchy, we would be able to find out the microscopic degrees of freedom which need to be introduced to describe general relativity at large space-time scales. Investigation along this line is now in progress and will be reported elsewhere.

\section{ACKNOWLEDGMENTS}

The authors thank Hikaru Kawai for useful discussions and Teiji Kunihiro for his nice lectures on relativistic fluid mechanics. This work was supported by the Grant-in-Aid for the Global COE program "The Next Generation of Physics, Spun from Universality and Emergence" from the Ministry of Education, Culture, Sports, Science and Technology (MEXT) of Japan. This work was also supported by the Japan Society for the Promotion of Science (JSPS) (Grant No. 21.1105) and by MEXT (Grant No. 19540288).

\section{APPENDIX: A DERIVATION OF THE ENTROPY FUNCTIONAL}

In this appendix, we derive the entropy functional (31) in the flat background, where the local entropy is assumed to take a simple form, $\tilde{s}(x)=\tilde{s}\left(\tilde{c}^{I}(x)\right)$. In the following, we consider the special cases where $N=1$ and $h_{i j}$ are constant.

As can be seen from the equilibrium condition (19), all intensive parameters $\beta_{I}$ are spatially constant in the equilibrium states. We take the region $\Sigma_{x}\left[L_{\mathrm{s}}\right]$ to be a $D$-dimensional torus with the coordinates $x^{i}$ having the period $L_{\mathrm{s}}$. We assume that finite size effects become irrelevant when $L_{\mathrm{s}} \gg \epsilon_{\mathrm{s}}$. Then the equilibrium values in $\Sigma_{x}\left[L_{\mathrm{s}}\right]$ are equal to the mean values of 
$c^{I}(x)$ in $\Sigma_{x}\left[L_{\mathrm{s}}\right]:$

$$
c_{0}^{I}=\frac{C^{I}}{V} \quad\left(V=\sqrt{h} L_{\mathrm{s}}^{D}\right) \quad \text { or } \quad \tilde{c}_{0}^{I}=\frac{C^{I}}{L_{\mathrm{s}}^{D}},
$$

where $C^{I}=\int d^{D} \boldsymbol{y} \tilde{c}^{I}(t, \boldsymbol{y})$ is the total charge for the region $\Sigma_{x}\left[L_{\mathrm{s}}\right]$. We Fourier-expand $\tilde{c}^{I}(t, \boldsymbol{y})$ as

$\tilde{c}^{I}(t, \boldsymbol{y})=\frac{1}{V} \sum_{\boldsymbol{k}} e^{i \boldsymbol{k} \cdot \boldsymbol{y}} \tilde{c}_{\boldsymbol{k}}^{I}(t)=\frac{C^{I}}{V}+\frac{1}{V} \sum_{\boldsymbol{k} \neq \boldsymbol{0}} e^{i \boldsymbol{k} \cdot \boldsymbol{y}} \tilde{c}_{\boldsymbol{k}}^{I}(t)$,

where $k_{i}=2 \pi n_{i} / L_{\mathrm{s}}\left(n_{i} \in \mathbb{Z}\right)$. The entropy functional

$$
\hat{S}\left(t ; \Sigma_{x}\left[L_{\mathrm{s}}\right]\right)=\int_{\Sigma_{x}\left[L_{\mathrm{s}}\right]} \tilde{s}\left[\tilde{c}^{I}(t, \boldsymbol{y})\right]
$$

can then be expanded as follows:

$$
\begin{aligned}
\hat{S}= & V s\left[\tilde{c}_{0}^{I}(t)\right]+\frac{1}{V} \int_{\Sigma_{x}\left[L_{\mathrm{s}}\right]} d^{D} \boldsymbol{y} \frac{\partial \tilde{s}}{\partial \tilde{c}^{I}}\left[\tilde{c}_{0}^{I}(t)\right] \sum_{\boldsymbol{k} \neq \mathbf{0}} e^{i \boldsymbol{k} \cdot \boldsymbol{y}} \tilde{c}_{\boldsymbol{k}}^{I}(t) \\
& +\frac{1}{2 V^{2}} \int_{\Sigma_{x}\left[L_{\mathrm{s}}\right]} d^{D} \boldsymbol{y} \frac{\partial^{2} \tilde{\boldsymbol{s}}}{\partial \tilde{c}^{I} \partial \tilde{c}^{J}}\left[\tilde{c}_{0}^{I}(t)\right] \\
& \times \sum_{\boldsymbol{k} \neq \mathbf{0}} \sum_{\boldsymbol{k}^{\prime} \neq \mathbf{0}} e^{i\left(\boldsymbol{k}+\boldsymbol{k}^{\prime}\right) \cdot \boldsymbol{y}} \tilde{c}_{\boldsymbol{k}}^{I}(t) \tilde{c}_{\boldsymbol{k}^{\prime}}^{J}(t) \\
= & \hat{S}_{0}+\frac{1}{2 V(\sqrt{h})^{2}} s_{I J}^{0} \sum_{\boldsymbol{k} \neq \mathbf{0}} \tilde{c}_{\boldsymbol{k}}^{I}(t) \tilde{c}_{-\boldsymbol{k}}^{J}(t),
\end{aligned}
$$

where $s_{I J}^{0}=\left.\sqrt{h}\left(\partial^{2} \tilde{s} / \partial \tilde{c}^{I} \partial \tilde{c}^{J}\right)\right|_{0}$, and we have used $\int d^{D} \boldsymbol{y} \sum_{\boldsymbol{k} \neq \boldsymbol{0}} e^{i \boldsymbol{k} \cdot \boldsymbol{y}} \tilde{c}_{\boldsymbol{k}}^{I}(t)=0$.

$\hat{S}-\hat{S}_{0}$ can be written as

$$
-\frac{1}{2 V(\sqrt{h})^{2}} \sum_{\boldsymbol{k}} f_{I J}(\boldsymbol{k}) \tilde{c}_{\boldsymbol{k}}^{I}(t) \tilde{c}_{-\boldsymbol{k}}^{J}(t)
$$

with $f_{I J}(\boldsymbol{k})=-s_{I J}^{0}\left(1-\delta_{\boldsymbol{k}, \mathbf{0}}\right)$. However, this nonanalytic form of $f_{I J}(\boldsymbol{k})$ is not desirable because it may change the longdistance behavior in the analysis based on the derivative expansion. The most desirable is such a function that filters out low- $\boldsymbol{k}$ modes analytically, and one can take as such the following function:

$$
f_{I J}(\boldsymbol{k})=\ell_{I, J}^{i j} k_{i} k_{j},
$$

where $\ell_{I, J}^{i j}$ is a positive definite tensor and is symmetric under the exchange $(i I) \leftrightarrow(j J)$. The (mild) increase of $f_{I J}(\boldsymbol{k})$ in the region $|\boldsymbol{k}| \gtrsim 2 \pi / L_{\mathrm{s}}$ does not cause a problem because $\tilde{c}_{\boldsymbol{k}}^{I}(t)$ are assumed to decrease rapidly in the same region. Then by using the equality $\partial_{i}\left(\partial \tilde{s} / \partial \tilde{c}^{I}\right)=(1 / \sqrt{h}) s_{I J} \partial_{i} \tilde{c}^{J}$, the entropy functional can be written as

$$
\begin{aligned}
\hat{S} & =\hat{S}_{0}-\frac{1}{2 V(\sqrt{h})^{2}} \sum_{\boldsymbol{k}} \ell_{I, J}^{i} k_{i} k_{j} \tilde{c}_{\boldsymbol{k}}^{I}(t) \tilde{c}_{-\boldsymbol{k}}^{J}(t) \\
& =\hat{S}_{0}-\frac{1}{2 \sqrt{h}} \int_{\Sigma_{x}\left[L_{\mathrm{s}}\right]} d^{D} \boldsymbol{y} \ell_{I, J}^{i j} \partial_{i} \tilde{c}^{I}(t, \boldsymbol{y}) \partial_{j} \tilde{c}^{J}(t, \boldsymbol{y}) \\
& =\hat{S}_{0}-\frac{\sqrt{h}}{2} \int_{\Sigma_{x}\left[L_{\mathrm{s}}\right]} d^{D} \boldsymbol{y} \ell^{i I, j J} \partial_{i}\left(\frac{\partial \tilde{s}}{\partial \tilde{c}^{I}}\right) \partial_{j}\left(\frac{\partial \tilde{s}}{\partial \tilde{c}^{J}}\right),
\end{aligned}
$$

where $\ell^{i I, j J} \equiv \ell_{K, L}^{i}{ }^{j}\left(s_{0}^{-1}\right)^{K I}\left(s_{0}^{-1}\right)^{L J}$. For a general metric, $\hat{S}$ will have the following form:

$$
\hat{S}=\hat{S}_{0}-\frac{1}{2} \int_{\Sigma_{x}\left[L_{\mathrm{s}}\right]} d^{D} \boldsymbol{x} N \sqrt{h} \ell^{\mu I, \nu J} \nabla_{\mu}\left(\frac{\partial \tilde{\boldsymbol{s}}}{\partial \tilde{c}^{I}}\right) \nabla_{\nu}\left(\frac{\partial \tilde{s}}{\partial \tilde{c}^{J}}\right),
$$

where the coefficients have only the spatial components, $\ell^{\mu I, v J} u_{v}=0$. If there further exist additional $b^{A}$ type variables, this functional will have the more general form (31).
[1] L. D. Landau and E. M. Lifshitz, Statistical Physics, Course of Theoretical Physics, 3rd ed., Part 1, Vol. 5 (ButterworthHeinemann, London, 1980).

[2] C. Eckart, Phys. Rev. 58, 919 (1940).

[3] L. D. Landau and E. M. Lifshitz, Fluid Mechanics, Course of Theoretical Physics, 2nd ed., Vol. 6 (Butterworth-Heinemann, London, 1987).

[4] N. Andersson and G. L. Comer, Living Rev. Relativ. 10, 1 (2007).

[5] P. Romatschke, Int. J. Mod. Phys. E 19, 1 (2010).

[6] L. Onsager, Phys. Rev. 37, 405 (1931).

[7] L. Onsager, Phys. Rev. 38, 2265 (1931).

[8] H. B. G. Casimir, Rev. Mod. Phys. 17, 343 (1945).

[9] S. R. de Groot and P. Mazur, Non-Equilibrium Thermodynamics (Dover, New York, 1984).
[10] C. Eckart, Phys. Rev. 73, 373 (1948).

[11] T. Azeyanagi, M. Fukuma, H. Kawai, and K. Yoshida, Phys. Lett. B 681, 290 (2009).

[12] T. Azeyanagi, M. Fukuma, H. Kawai, and K. Yoshida, to appear Proceedings of Quantum Theory and Symmetries 6 (2011), e-print arXiv:1004.3899 [hep-th].

[13] R. L. Arnowitt, S. Deser, and C. W. Misner, Gravitation: An Introduction to Current Research, edited by Louis Witten, Chap. 7 (Wiley, New York, 1962), pp. 227-265.

[14] L. D. Landau and E. M. Lifshitz, Theory of Elasticity, Course of Theoretical Physics, 3rd ed., Vol. 7 (Butterworth-Heinemann, London, 1986).

[15] M. Fukuma and Y. Sakatani, Phys. Rev. E 84, 026316 (2011).

[16] M. Fukuma and Y. Sakatani (in preparation). 